\title{
2 Rice Phenology Mapping Using Novel Target Characterization 3 Parameters from Polarimetric SAR Data
}

\author{
Subhadip Dey ${ }^{\mathrm{a}}$ (D), Narayanarao Bhogapurapu ${ }^{\mathrm{a}}$ (D), Avik Bhattacharya ${ }^{\mathrm{a}}$ \\ Dipankar Mandal $^{\mathrm{a}}$ (D), Juan M. Lopez-Sanchez ${ }^{\mathrm{b}}$ (D), Heather McNairn ${ }^{\mathrm{c}}$ (D) and \\ Alejandro C. Frery ${ }^{\mathrm{d}}$ (D) \\ ${ }^{a}$ Microwave Remote Sensing Lab, Centre of Studies in Resources Engineering, Indian \\ Institute of Technology Bombay, Mumbai, India; \\ ${ }^{b}$ Institute for Computer Research, University of Alicante, Alicante, Spain; \\ ${ }^{\mathrm{c}}$ Ottawa Research and Development Centre, Agriculture and Agri-Food Canada, Ottawa, \\ 11 Canada; \\ $12{ }^{\mathrm{d}}$ School of Mathematics and Statistics, Victoria University of Wellington, New Zealand
}

\section{ARTICLE HISTORY \\ Compiled April 22, 2021}

\begin{abstract}
We require Spatio-temporal information about rice for executing and planning diverse management practices. In this regard, data obtained from Synthetic Aperture Radar (SAR) sensors are well suited for tracking morphological developments of rice across its phenology stages. This study proposes different target characterization parameters from polarimetric SAR data for rice phenology mapping. Six C-band Radarsat-2 images acquired over Vijayawada, India, are used for complete analysis. It is known that polarimetric information provides excellent sensitivity for identifying crop phenology stages. Hence, in this study, we assessed phenology classification results using a scattering-type parameter and scattering powers for full-polarimetric (FP) and extracted dual-polarimetric (DP) SAR data. Here, we utilized the real $4 \times 4$ Kennaugh matrix elements to derive these parameters equivalently for the two polarimetric modes (i.e., FP and DP). We obtained better overall classification accuracy for each phenology stages using the proposed parameters than the existing ones from FP and DP SAR data. We noted that the overall classification accuracy using the DP SAR data was only marginally lower than the FP SAR data. This marginal difference in the accuracies could be due to the absence of the cross-polarized component in the DP SAR data. We also demonstrate the usefulness of the scattering powers from DP SAR data for rice phenology monitoring.
\end{abstract}

\section{KEYWORDS}

Supervised classification; Dual Co-pol; Radarsat-2; Phenology mapping; Full polarimetry; Model-free decomposition; Information content

CONTACT Subhadip Dey. Email: sdey2307@gmail.com Microwave Remote Sensing Lab, Centre of Studies in Resources Engineering, Indian Institute of Technology Bombay, Mumbai, India 


\section{Introduction}

61

Rice is an important global crop and is a staple food grown in the Indian subcontinent. Having the requirement of large volumes of water, rice cultivation practice is generally concentrated in moderate to high rainfed areas. Throughout its cultivation period, it is essential to monitor several critical phenological stages to maximize rice productivity. These critical phenological stages include tillering, flowering and grain filling periods (Lampayan et al. 2015, Mahajan, Bharaj, and Timsina 2009). Hence, it is vital to monitor the dynamics of rice growth during these phenological stages over large production regions.

Synthetic Aperture Radar (SAR) data provide valuable information to characterize the development of rice canopies at different phenological stages. Previous studies have obtained good results for rice monitoring and mapping using X-band Corcione et al. 2016: Yuzugullu, Erten, and Hajnsek 2015, Küçük, Taşkın, and Erten 2016, De Bernardis et al. 2015, Erten et al. 2016, Koppe et al. 2013), and C-band sensors (Zhang et al. 2014, Bouvet, Le Toan, and Lam-Dao 2009, Lopez-Sanchez et al. 2014, Yang et al. 2017; Tian et al. 2018). The radar backscattering coefficient has proven to be a good indicator of rice phenological changes. Le Toan et al. (1989) utilized dual-polarized C-band airborne SAR data to investigate the temporal backscatter response $\left(\sigma_{\mathrm{HH}}^{0}\right.$ and $\left.\sigma_{\mathrm{VV}}^{0}\right)$ from rice fields. Temporal variations of $\sigma_{\mathrm{HH}}^{0}$ and $\sigma_{\mathrm{VV}}^{0}$ assisted in mapping rice fields according to the time of sowing and phenological stages. Similarly, radar backscatter changes over time are indicative of the phenological development of rice (Kurosu, Fujita, and Chiba 1995).

Several other studies confirmed that the temporal response of co-polarized (i.e., $\mathrm{HH}$ and VV) backscatter coefficients aptly capture the phenological evolution of rice (Le Toan et al. 1997; Koay et al. 2007; Bouvet, Le Toan, and Lam-Dao 2009). 
More recently, Torbick et al. (2017) utilized the VV and VH backscatter coefficients of Sentinel-1 C-band SAR data to map the spatial variation of phenological stages for rice producing regions of Myanmar. Backscatter values for a given polarization are useful for inferring structural information of a target, but offer limited understanding of the diverse scattering mechanisms. Such features describe the complex interactions of the electromagnetic (EM) wave between the soil and crop and within the canopy itself. In this respect, often, scattering mechanisms are often distinguished by different scattering power decomposition techniques.

Freeman and Durden (1998) paved the way for model-based decomposition techniques by introducing a three-component scattering power decomposition. Later Yamaguchi et al. (2011) proposed the four-component approach incorporating the helix scattering model as a fourth component. Furthermore, Cloude and Pottier (1997) and Touzi (2006) decomposed the average covariance or coherency matrices with eigenvalue-eigenvector based decomposition techniques to provide a unique solution to discriminate scattering mechanisms. These decomposition methods have received considerable attention over the last decade. Li et al. (2012) utilized the Freeman-Durden, Cloude-Pottier, and the Touzi decomposition parameters to map and monitor rice crops. The study showed that the decomposition parameters enhanced the ability to monitor rice due to changes in the scattering phase centre with plant maturity. The Freeman-Durden decomposition produced the highest classification accuracy (83\%) for rice identification, compared to the Touzi $(82 \%)$ and the Cloude-Pottier (80\%) decompositions.

Lopez-Sanchez et al. (2014) investigated the differential variations of the scattering power components from the Freeman-Durden decomposition with rice growth stages. Unique signatures obtained from these scattering powers at each growth stages help 
to identify rice phenology. However, the Freeman-Durden decomposition power components are sensitive to the orientation of targets, and the volume scattering model uses an ensemble of uniformly distributed dipoles. Therefore, this particular volume model assumption may be seldom valid for all phenology stages for different crops.

a Lopez-Sanchez, Ballester-Berman, and Hajnsek (2012), and Lopez-Sanchez, Cloude, and Ballester-Berman (2012) investigated the importance of the dual co-polarized TerraSAR-X data for monitoring rice phenological stages. The phase and correlation between the two channels ( $\mathrm{HH}$ and $\mathrm{VV}$ ) provided valuable information with respect to crop growth development. In their study, the Cloude $\alpha$ parameter from the dual copol X-band SAR data showed significant sensitivity with rice development, where the scattering mechanisms distinctly responded to phenological changes. Lopez-Sanchez, Cloude, and Ballester-Berman (2012) introduced a model-based decomposition technique for dual co-polarimetric SAR data. The decomposition technique estimates a random volume component plus a polarized contribution of the scattered wave (a rank-1 mechanism). This technique provides useful polarimetric information on rice morphology at different phenological stages.

In a separate study, Ullmann, Schmitt, and Jagdhuber (2016) proposed a twocomponent decomposition technique for dual co-pol data. The approach is adapted from the Yamaguchi decomposition. The data is decomposed into two scattering contributions: surface and double-bounce under the assumption of a negligible vegetation scattering component in the Tundra environment. Hence, this decomposition technique might not provide adequate information for diffused, or the volume scattering component for crops. In another study, Dey et al. (2020a) proposed unsupervised clustering schemes for full and compact polarimetric SAR data using the target scattering-type parameter (Dey et al. 2020). Their study utilized this scattering-type parameter along 
with the scattering entropy to characterize different phenological stages of rice. The work highlighted the advantages of the novel clustering schemes for phenology classification for both full and compact polarimetric SAR data.

In the existing dual co-pol decomposition techniques proposed by Ullmann, Schmitt, and Jagdhuber (2016) and Lopez-Sanchez, Cloude, and Ballester-Berman (2012), the power components are either: (1) two polarized power components, or (2) a volume plus a polarized power component. Therefore, a target in an image can not be uniquely characterized using these techniques. In addition, none of these methods consider a measure of the degree of polarization of the scattered wave. The degree of polarization essentially indicates the total polarized scattered component from a target. Thus, it may provide additional information about the morphology of the target.

In this study, we propose a model-free scattering power decomposition framework uniformly for full-polarimetric (FP) and dual co-polarimetric (DP) SAR data. The technique utilizes information about the polarization state of the scattered wave in terms of the Barakat degree of polarization (Barakat 1977) and the Kennaugh matrix elements. Moreover, the scattering-type parameters for both FP and DP SAR data obtained from this technique are roll-invariant (i.e., independent of the target orientation angle about the radar line of sight).

We use these power components to classify different phenological stages of rice. The phenology classification accuracy is compared with scattering powers obtained from other existing decomposition techniques. In summary, this study focuses on:

- Proposal of a framework utilizing the Kennaugh matrix to describe the novel target scattering-type parameter from FP and DP SAR data.

- Utilization of this parameter in the proposal of a novel model-free 3 component scattering power decomposition for DP SAR data. 
- Application of the scattering-type parameter and the power components for rice phenology classification.

- Comparison of the classification accuracy utilizing the DP parameters with FP SAR data.

\section{Study area}

The study area is located near the city of Vijayawada in the state of Andhra Pradesh, India $\left(16^{\circ} 24^{\prime} 6.2^{\prime \prime} \mathrm{N}, 8^{\circ} 41^{\prime} 2.4^{\prime \prime} \mathrm{E}\right)$ as shown in Figure 1 (Mandal et al.|2019). The climatic zone varies from sub-humid to humid within the study area, with mostly clayey soil texture in this region. The spatial coverage of this test site is $\approx 25 \mathrm{~km} \times 25 \mathrm{~km}$. Rice is a major crop grown in this area. Depending on the variety and cultivation practices, the sowing period of rice varies from mid-June to mid-July. However, in general, cultivation starts after the pre-monsoon rain and rice is harvested during mid-December. The average field size is $\approx 60 \mathrm{~m} \times 60 \mathrm{~m}$. In each field, two sampling locations were chosen for in-situ measurements. Information about the crop growth stage, management practices, and biophysical parameters was noted during a field campaign which was conducted from June to December 2019.

[Figure 1 about here.]

\section{Satellite data sets and pre-processing}

55 Fine Quad Wide (FQW) mode Radarsat-2 images were acquired from July to November 2019 over the test site, as shown in Table 1. These images were multilooked by a factor of $2 \times 3$ pixels in the range and azimuth directions, respectively, to generate $\approx 15 \mathrm{~m} \times 15 \mathrm{~m}$ square pixel images. During rice cultivation typically many adjacent 
fields are cultivated together. Therefore, the region appears homogeneously cropped, even though each individual parcel's size is small. Hence, a $3 \times 3$ boxcar filter (Lee and Pottier 2009) was applied to reduce speckle. Dual co-polarimetric (DP) SAR data (HH-VV) were extracted from the FP data and are subsequently co-registered with a Root Mean Square Error (RMSE) $\leq 0.25 \mathrm{~m}$.

[Table 1 about here.]

\section{In situ measurement procedures}

In this study, 40 in-situ field measurements were analyzed. Soil moisture was measured in each field for two sampling locations. These locations were arranged in two parallel transects along the row direction, with a separation between each transect of $\approx 40 \mathrm{~m}$. Theta-probes were used to collect moisture measurements. The soil was saturated during the majority of the season due to irrigation and rainfall events. Vegetation measurements were gathered at two points in each field. The location of these points was driven by the spatial heterogeneity of plant growth within the fields. At each point, the Plant Area Index (PAI), plant height, and phenology were measured using non-destructive methods. The PAI was determined using hemispherical digital photography, with ten photos collected at each sample point along two transects separated by $2 \mathrm{~m}$. Photographs were taken using a wide-angle lens mounted on a digital camera. All photos were post-processed using the CanEYE software (https://www6.paca.inra.fr/can-eye), which calculates an estimate of the PAI. Descriptions of distinct phenology stages of rice are shown in Table 2 and the statistics of different bio-physical and soil parameters are given in Table 3 .

[Table 2 about here.] 
The morphological developments of rice across its various phenological stages are shown in Figure 2. The growth stages of rice usually consist of three major phases: vegetative, reproductive, and maturity. In the test site, the cultivation time of rice depends mainly on rainfall events. The growing period of rice varies from 100 days to 140 days, depending on the varieties of rice. Short-duration cultivation lasts for 100-120 days, medium-duration for 120-140 days, and long-duration for almost 160 days (Adhikari et al. 2011). The vegetative phase begins with the germination of the seeds and ends with fully developed plants. In this period, the number of tillers increases and stems elongate. The germination phase consists of seed and radicle development. The onset of the first tiller defines the initiation of the tillering stage. During booting, the upper part of the stem becomes thick, and flag leaf development begins. During this period, the majority of plants remain vertical, known as the erectophile structure. Subsequently, panicle emergence and heading stages are observed. During this period, significant leaf inclination and increased random structures are visible. The fruit development stage starts when the grain appears and later becomes milky following the complete development of anthers. In the subsequent stage, dough followed by the ripening condition of rice leads to the final harvest. During the late-ripening period, the plant-water content drops, and rice appears to be dry. The fully grown structure of rice becomes random as grains become heavy, while the number of leaves decreases (Moldenhauer and Slaton 2001).

[Figure 2 about here.] 


\section{Methodology}

In polarimetric SAR, the $2 \times 2$ complex scattering matrix $\mathbf{S}$ contains complete polarimetric information about backscattering from targets for each pixel. It is expressed in the backscatter alignment (BSA) convention in the linear horizontal $(\mathrm{H})$ and linear vertical (V) polarization basis as,

$$
\mathbf{S}=\left[\begin{array}{cc}
S_{\mathrm{HH}} & S_{\mathrm{HV}} \\
S_{\mathrm{VH}} & S_{\mathrm{VV}}
\end{array}\right] \Rightarrow \boldsymbol{k}=V([\mathbf{S}])=\frac{1}{2} \operatorname{tr}(\mathbf{S} \Psi)
$$

where $V(\cdot)$ is the vectorization operator on the scattering matrix, $\Psi$ is the corresponding basis matrix, and tr is the sum of the diagonal elements of the matrix. Each element of the matrix represents the backscattering response of the target at a specific polarization. The matrix's diagonal elements represent the co-polarized scattering information, while the off-diagonal terms represent the cross-polarized information. In the monostatic backscattering case, the reciprocity theorem constrains the scattering matrix to be symmetric (i.e., $S_{\mathrm{HV}}=S_{\mathrm{VH}}$ ).

The multi-looked Hermitian positive semi-definite $3 \times 3$ coherency matrix $\mathbf{T}$ is obtained from the averaged outer product of the target vector $\boldsymbol{k}_{\mathrm{P}}$ (derived using the Pauli basis matrix, $\Psi_{\mathrm{P}}$ ) with its conjugate (i.e., $\mathbf{T}=\left\langle\boldsymbol{k}_{\mathrm{P}} \cdot \boldsymbol{k}_{\mathrm{P}}^{* \mathrm{~T}}\right\rangle$ ). Similarly, the $3 \times 3$ covariance matrix $\mathbf{C}$ is obtained from the averaged outer product of the target vector $\boldsymbol{k}_{\mathrm{L}}$ (derived using the Lexicographic basis matrix, $\Psi_{\mathrm{L}}$ ) with its conjugate (i.e., 
$\left.\mathbf{C}=\left\langle\boldsymbol{k}_{\mathrm{L}} \cdot \boldsymbol{k}_{\mathrm{L}}^{* \mathrm{~T}}\right\rangle\right)$

$$
\begin{aligned}
& \Psi_{\mathrm{P}}=\left\{\sqrt{2}\left[\begin{array}{ll}
1 & 0 \\
0 & 1
\end{array}\right] \quad \sqrt{2}\left[\begin{array}{cc}
1 & 0 \\
0 & -1
\end{array}\right] \quad \sqrt{2}\left[\begin{array}{ll}
0 & 1 \\
1 & 0
\end{array}\right]\right\}, \\
& \Psi_{\mathrm{L}}=\left\{2\left[\begin{array}{ll}
1 & 0 \\
0 & 0
\end{array}\right] \quad 2 \sqrt{2}\left[\begin{array}{ll}
0 & 1 \\
0 & 0
\end{array}\right] \quad 2\left[\begin{array}{ll}
0 & 0 \\
0 & 1
\end{array}\right]\right\} .
\end{aligned}
$$

$$
k_{i j}=\frac{1}{2} \operatorname{tr}\left(\mathbf{T} \eta_{4 i+j}\right), \quad \text { where } \quad \eta_{4 i+j}=(-1)^{\left(-\delta_{3 j}\right)} \mathbf{A}\left(\sigma_{i} \otimes \sigma_{j}\right) \mathbf{A}^{* \mathrm{~T}}
$$

where $k_{i j}$ is an elements of $\mathbf{K}$, each $\sigma_{i}$ is one of the four Pauli basis matrices and * represents the complex conjugate. Here, $\eta$ is a set of sixteen $4 \times 4$ matrices (the generators of the group $\mathrm{SU}(4)$ plus the unit matrix) indexed by $i$ and $j$ as the elements

of $\mathbf{K}$. These matrices represent a generalization of the Pauli matrices in $\mathbb{C}^{4}$, i.e., the 4-dimensional complex space $($ Cloude 1986$)$. The $4 \times 4$ matrix formed from the four 223 Pauli vectors is the unitary matrix $\mathbf{A}$ given as,

$$
\mathbf{A}=\frac{1}{\sqrt{2}}\left[\begin{array}{cccc}
1 & 0 & 0 & 1 \\
1 & 0 & 0 & -1 \\
0 & 1 & 1 & 0 \\
0 & i & -i & 0
\end{array}\right], \text { and } \delta_{i j}=\left\{\begin{array}{cc}
0 & i \neq j \\
1 & i=j
\end{array}\right.
$$

224 where $\delta_{i j}$ is the Kronecker delta function indexed by $i$ and $j$. Like the conventional 225 degree of polarization (Born and Wolf 2013), the $n$ D Barakat degree of polarization $m_{n}$ 
similarly characterizes the state of polarization (or purity) of an EM wave described by the $n \times n$ coherency matrix $\mathbf{T}$ (Barakat 1977). The expression of $m_{n}$ is given as,

$$
m_{n}=\sqrt{1-\frac{n^{n}|\mathbf{T}|}{\operatorname{tr}^{n}(\mathbf{T})}}, \quad 0 \leq m_{n} \leq 1
$$

In this study, $\mathbf{T}$ is either the $3 \times 3$ or the $2 \times 2$ coherency matrix for $\mathrm{FP}(\mathrm{HH}|\mathrm{HV}| \mathrm{VH} \mid \mathrm{VV})$ or DP (HH|VV) SAR data, respectively. It can be shown that the Barakat degree of polarization is linked to the polarimetric contribution of the Shannon entropy (Réfrégier et al. 2004). Here the subscript (or superscript) $n$ denotes either FP or DP SAR data depending on the coherency matrix dimension.

In order to derive the scattering-type parameter $\theta_{n}$ from the FP or DP SAR data, let us first consider two free variables, $\eta_{1}$ and $\eta_{2}$ as,

$$
\eta_{1}=\tan ^{-1}\left(\frac{k_{11}-k_{44}}{2 m_{n} k_{11}}\right) \quad \text { and } \quad \eta_{2}=\tan ^{-1}\left(\frac{k_{11}+k_{44}}{2 m_{n} k_{11}}\right)
$$

that $\frac{k_{11}-k_{44}}{2 m_{n} k_{11}}$ denotes the fraction of power scattered from the regular part of a target with respect to the total polarized power, and $\frac{k_{11}+k_{44}}{2 m_{n} k_{11}}$ denotes the fraction of scattered power from the irregular part of a target with respect to the total polarized power (Huynen 1970). Hence, by using a simple relationship, we obtain,

$$
\begin{aligned}
\tan \theta_{n} & =\tan \left(\eta_{1}-\eta_{2}\right) \\
& =\frac{4 m_{n} k_{11} k_{44}}{k_{44}^{2}-\left(1+4 m_{n}^{2}\right) k_{11}^{2}}
\end{aligned}
$$


and $k_{11}=\left(T_{11}+T_{22}\right) / 2$ and $k_{44}=\left(-T_{11}+T_{22}\right) / 2$ for DP SAR data. $T_{i i}$ for $i=1,2,3$ are the diagonal elements of $\mathbf{T}$.

Utilizing $\theta_{n} \in\left[-45^{\circ}, 45^{\circ}\right]$, we split the polarized part of the total power, i.e., $2 m_{n} k_{11}$, into two components: even-bounce $\left(P_{\mathrm{d}}^{n}\right)$, and odd-bounce $\left(P_{\mathrm{s}}^{n}\right)$ scattering powers using a geometrical factor $\left(1 \pm \sin 2 \theta_{n}\right)$. The diffused (i.e., depolarized) scattering power, $\left(P_{\mathrm{v}}^{n}\right)$ is obtained as the depolarized part of the total power:

$$
\begin{aligned}
& P_{\mathrm{d}}^{n}=m_{n} k_{11}\left(1-\sin 2 \theta_{n}\right), \\
& P_{\mathrm{v}}^{n}=2 k_{11}\left(1-m_{n}\right), \text { and } \\
& P_{\mathrm{s}}^{n}=m_{n} k_{11}\left(1+\sin 2 \theta_{n}\right) .
\end{aligned}
$$

Variations of $\left(1-\sin 2 \theta_{n}\right)$ and $\left(1+\sin 2 \theta_{n}\right)$ for $\theta_{n} \in\left[-45^{\circ}, 45^{\circ}\right]$ is shown in Figure 3 . The two extremes of $\theta_{n}$ are labelled as $P_{\mathrm{s}}$ and $P_{\mathrm{d}}$. It can be noted from Figure $3(\mathrm{a})$ that for $\left(1+\sin 2 \theta_{n}\right): P_{\mathrm{s}}=2.0$ and $P_{\mathrm{d}}=0.0$. These values indicate that $\left(1+\sin 2 \theta_{n}\right)$ characterizes the odd-bounce scattering component. In contrast, it can be noted from Figure 3(b) that for $\left(1-\sin 2 \theta_{n}\right): P_{\mathrm{s}}=0.0$ and $P_{\mathrm{d}}=2.0$. These values indicate that $\left(1-\sin 2 \theta_{n}\right)$ characterizes the even-bounce scattering component.

[Figure 3 about here.]

The following special cases are of particular interest:

Complete depolarization: $m_{n}=0$, then $P_{\mathrm{d}}^{n}=P_{\mathrm{s}}^{n}=0$, and $P_{\mathrm{v}}^{n}=2 k_{11}$.

Pure even-bounce: $m_{n}=1$, and $\theta_{n}=-45^{\circ}$ with $P_{\mathrm{s}}^{n}=P_{\mathrm{v}}^{n}=0$, and $P_{\mathrm{d}}^{n}=2 k_{11}$.

Pure odd-bounce: $m_{n}=1$, and $\theta_{n}=45^{\circ}$ with $P_{\mathrm{d}}^{n}=P_{\mathrm{v}}^{n}=0$, and $P_{\mathrm{s}}^{n}=2 k_{11}$.

Therefore, the advantages of this proposed decomposition technique can be summarized as: 
- The proposed decomposition technique is model-free, i.e., no prior assumption on the type of scatterer existing within the scene is assumed. This is unlike the conventional model-based decomposition techniques in the literature.

- It can be noted that the scattering-type parameter $\theta_{n}$, along with the scattering powers, $P_{\mathrm{d}}^{n}, P_{\mathrm{s}}^{n}$ and $P_{\mathrm{v}}^{n}$ are roll-invariant (i.e., independent of target orientation angle about the radar line of sight).

- The scattering power components are non-negative and the total power $\left(2 k_{11}\right)$ is conserved for any polarization state

For the sake of convenience, in the following text, we address the proposed FP decomposition as MF3CF and the DP decomposition as MF3CD.

\section{Results and Discussion}

This section presents the temporal analysis for diverse rice growth stages using the MF3CF and MF3CD decomposition parameters obtained from the Radarsat-2 fullpol and extracted dual co-pol dataset. Figures 4 and 8 show the temporal variation of $\theta_{\mathrm{FP}}$ and $\theta_{\mathrm{DP}}$ for different phenology stages of rice in this region. These values of $\theta_{\mathrm{FP}}$ and $\theta_{\mathrm{DP}}$, along with the scattering power components, are used for supervised classification of rice phenological stages (as shown in Figures 7 and 11). We used the bootstrap sampling procedure with 600 trees with a maximum depth of 10 trees for classification using the random forest $(\mathrm{RF})$ technique. The out of bag error starts to saturate beyond 600 trees. Several studies reported in the literature (Lawrence, Wood, and Sheley 2006; Dey et al. 2020b) confirms this fact. Tables 4 and 6 present an analysis of the clustering accuracies. The confusion matrix for full pol is given in Figures 6(a), 6(b), 6(c), 6(d), and for dual pol is given in Figures 10(a), 10(b), 10(c), 
To compare the effectiveness of MF3CD's powers for classification, we first conduct its analysis with MF3CF's powers, proposed in Dey et al. (2020) for FP SAR data.

[Figure 4 about here.]

\subsection{Full-polarimetric SAR data}

Utilizing MF3CF, we compute the scattering-type parameter, $\theta_{\mathrm{FP}}$ from the fullpolarimetric C-band Radarsat-2 SAR data. The spatial variation of $\theta_{\mathrm{FP}}$ as a function of rice phenology is shown in Figure 4 . In total, 4 sample fields (F1, F2, F3 and F4) are used for the trend analysis of $\theta_{\mathrm{FP}}$ (Figure 5). The temporal trend in $\theta_{\mathrm{FP}}, P_{\mathrm{d}}^{\mathrm{FP}}$, $P_{\mathrm{s}}^{\mathrm{FP}}$ and $P_{\mathrm{v}}^{\mathrm{FP}}$ indicates phenological development of rice.

At the beginning of the cultivation season, most of the fields were bare and smooth. Hence, on 06 June $2019, \theta_{\mathrm{FP}}$ ranges between $\left[30^{\circ}, 40^{\circ}\right]$, which indicates odd-bounce scattering, with some variations due to differences in surface roughness in different fields.

The degree of polarization $\left(m_{\mathrm{FP}}\right)$ during this period is also high and varies between 0.7 to 0.9 . These high values are due to the fairly smooth field condition at this early stage. Hence, predominant odd-bounce scattering power is evident from Figure 5 . Therefore, $P_{\mathrm{s}}^{\mathrm{FP}}$ is the dominant scattering power that varies $\approx 70 \%$ to $80 \%$.

In contrast, $P_{\mathrm{d}}^{\mathrm{FP}}$ and $P_{\mathrm{v}}^{\mathrm{FP}}$ values are fairly low early in this season. These low values of the scattering power components obtained from MF3CF are due to the absence of crop structure and a high coherent scattering mechanism. However, an anomaly in the values of $\theta_{\mathrm{FP}}, m_{\mathrm{FP}}$ and $P_{\mathrm{s}}^{\mathrm{FP}}$ is observed in field F3. The exception in the values of these two parameters might be due to the moderate roughness condition, which occurred due to ploughing.

On 24 July 2019, rice entered the early tillering stage. With the onset of rice mor- 
phology, $\theta_{\mathrm{FP}}$ begin to decrease. At this stage, the values of $\theta_{\mathrm{FP}}$ are $\approx 15^{\circ}$ for most of the fields. These low values of $\theta_{\mathrm{FP}}$ are due to foliage growth, which must have slightly increased randomness in the scattering phenomenon.

However, for field F2, it is observed that the value of $\theta_{\mathrm{FP}}$ is $\approx 0^{\circ}$, i.e., the high amount of randomness present in the scattered wave. Rice growth in this field was more advanced. As a result the large number of tillers may explain this low value of $\theta_{\mathrm{FP}}$. The PAI was around $0.9 \mathrm{~m}^{2} \mathrm{~m}^{-2}$ during this active tillering stage.

The increase in scattering randomness further affects the overall polarization structure of the backscattered wave. The degree of polarization is lower as compared to 06 June 2019. During this period, $m_{\mathrm{FP}}$ varies between 0.6 to 0.8 depending on the growth characteristics in different rice fields. Although the values of $m_{\mathrm{FP}}$ are low, the crops were still sparsely distributed. Hence, the scattering from the water surface beneath the crops was dominant during this period. As a consequence, this high backscatter from the water surface leads to a dominant $P_{\mathrm{s}}^{\mathrm{FP}}$ power component.

On the other hand, the interaction of the wave with crop stems and the water surface generated a small increment in the $P_{\mathrm{d}}^{\mathrm{FP}}$ power component. Besides, the amount of diffused power component has also increased substantially due to the emergence and development of the crops. During this period, the proportion of $P_{\mathrm{s}}^{\mathrm{FP}}$ power was $\approx 60 \%$, while the proportion of $P_{\mathrm{d}}^{\mathrm{FP}}$ and $P_{\mathrm{v}}^{\mathrm{FP}}$ powers were $\approx 16 \%$ and $\approx 24 \%$, respectively. $\theta_{\mathrm{FP}}$ continues to decrease on 17 August 2019 when the majority of rice fields were in their advanced tillering stage. At this stage, the values of $\theta_{\mathrm{FP}}$ are $\approx-16^{\circ}$, which indicates a change in the scattering mechanisms. This shift in the scattering mechanism is mainly due to the emergence and development of rice plants. The appearance of evenbounce scattering is due to the combined backscattering effect from the crop stem and the underlying water surface. 
During this period, the vertical structure of the crop and the underlying water jointly enhanced the degree of polarization of the scattered wave. However, differences in the trend of $m_{\mathrm{FP}}$ are observed among F1, F2, F3 and F4 fields. A slight increase in the values of $m_{\mathrm{FP}}$ is evident for F1 and F3 fields from Figure 5, while a decrease is observed for fields $\mathrm{F} 2$ and $\mathrm{F} 4$. The increase in $m_{\mathrm{FP}}$ for $\mathrm{F} 1$ and F3 fields is likely due to the development of prominent vertical crop structure. In contrast, the decrease for F2 and F4 fields might be due to the non-homogeneous growth of the plants, where some canopies were more advanced than others.

Rice was cultivated 4-6 days earlier in the F2 and F4 fields than the F1 and F3 fields. Hence, the canopy cover in fields F2 and F4 was higher relative to F1 and F3. Nevertheless, the crop growth in each field, except F2 has led to a dominance of $P_{\mathrm{d}}^{\mathrm{FP}}$ power during scattering.

At this stage of development, the value of $P_{\mathrm{d}}^{\mathrm{FP}}$ is $\approx 53 \%$, while the value of $P_{\mathrm{v}}^{\mathrm{FP}}$ is $\approx 26 \%$. In field F2, the complex crop geometry might have produced a high amount of diffused power component. It is important to note that from the date onwards, $P_{\mathrm{v}}^{\mathrm{FP}}$ shows an increasing trend for most fields.

The remaining phenological stages are dominated by even-bounce scattering, due to the interaction of the wave with stems and the water surface. This feature was earlier reported by Lopez-Sanchez et al. (2014) while analyzing Cloude alpha and the phase difference between the $\mathrm{HH}$ and VV channels.

During this period, some fields showed variations in $\theta_{\mathrm{FP}}$ between $-5^{\circ}$ and $10^{\circ}$ due to leaf and foliage development. These stems and leaves have increased scattering randomness, which is well characterized by $\theta_{\mathrm{FP}}$ as multiple bounce effects.

Like 17 August 2019, a variation in the values of $m_{\mathrm{FP}}$ is observed depending upon 
the crop morphological conditions. The complex structure of the leaves and foliage might have also increased the diffused power component, $P_{\mathrm{v}}^{\mathrm{FP}}$. However, the $P_{\mathrm{d}}^{\mathrm{FP}}$ power is yet dominant due to the interaction of the electromagnetic wave with the stem and water.

In particular, significant multiple bounces are observed during the advanced reproductive stage of rice. At this time, the emergence of flowers contribute to a more random crop geometry. Hence, on 04 October $2019, \theta_{\mathrm{FP}}$ varies within $\approx\left[-14^{\circ}, 14^{\circ}\right]$. During this period, although the $P_{\mathrm{d}}^{\mathrm{FP}}$ power is slightly higher than other power components, a shift in the dominancy between $P_{\mathrm{d}}^{\mathrm{FP}}$ and $P_{\mathrm{v}}^{\mathrm{FP}}$ powers begins during this stage of growth.

The amount of $P_{\mathrm{d}}^{\mathrm{FP}}$ power is $40 \%$ to $60 \%$, while the $P_{\mathrm{v}}^{\mathrm{FP}}$ power is $20 \%$ to $40 \%$. As the crop matures on 21 November $2019, \theta_{\mathrm{FP}}$ is centred on $0^{\circ}$. The dominance of $P_{\mathrm{v}}^{\mathrm{FP}}$ power is evident for all fields, and $m_{\mathrm{FP}}$ decreases due to an increase in scattering randomness. $\theta_{\mathrm{FP}}$ fluctuates around $0^{\circ}$, with the exception of field F4. During this period, most crop stems start to incline, although for some fields the crops remain vertical and the stem-water interaction generates a moderate amount of even-bounce power. At this time, the proportion of $P_{\mathrm{v}}^{\mathrm{FP}}$ power is $40 \%$ to $60 \%$, while $P_{\mathrm{d}}^{\mathrm{FP}}$ and $P_{\mathrm{s}}^{\mathrm{FP}}$ powers are $\approx 30 \%$ and $\approx 20 \%$, respectively.

We used these three scattering power components, $P_{\mathrm{s}}^{\mathrm{FP}}, P_{\mathrm{d}}^{\mathrm{FP}}$ and $P_{\mathrm{v}}^{\mathrm{FP}}$ to classify different phenological stages of rice with the multi-temporal C-band Radarsat-2 SAR dataset. In this analysis, we used the powers obtained from the An3D decomposi-

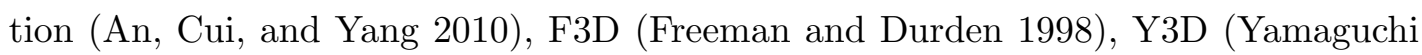
3-component decomposition) techniques and compared the classification with the powers obtained from the proposed FP method.

[Figure 6 about here.] 
The phenology classification accuracies with the RF classifier are provided in Table 4 using the MF3CF, An3D, F3D and Y3D three-component full-polarimetric decomposition techniques. We have particularly limited the classification comparison with conventional three-component decomposition techniques since the proposed decomposition technique generates only three scattering power components.

Table 5 details the global measures aggregated from Table 4. It can be noticed from Table 4, that the producer's accuracy (PA) for all bare fields using MF3CF is $100 \%$. In contrast, the PA using F3D, Y3D and An3D are $85.41 \%, 89.61 \%$ and $79.17 \%$, respectively. This misclassification might be due to marginal confusion arising between bare field condition, early tillering and booting stages which is evident from Figure 6(a), Figure 6(c) and Figure 6(b) . The assumptions of particular volume models in the model-based decomposition techniques might have raised the marginal confusion among the phenological stages. During this period, significant odd-bounce and volume scattering power components might have increased the confusion among these stages.

The user's accuracy (UA) using MF3CF is slightly low (94.11\%). This indicates that other phenology classes are misclassified as a bare field class (Figure 6(d)). As a consequence, Figure 7 maps the majority of the fields as bare, while a few fields are more phenologically advanced depending upon date of sowing.

[Table 4 about here.]

[Table 5 about here.]

During early and advanced tillering stages, the PA drops to $82.92 \%$ and $86.11 \%$, respectively. This decrease might be due to the similarity in the scattering mechanisms and the power components between the early tillering stages and advanced tillering. 
A similar reduction in $\mathrm{PA}$ is also evident for $\mathrm{F} 3 \mathrm{D}$, An3D and $\mathrm{Y} 3 \mathrm{D}$.

The similarity between scattering mechanisms and the corresponding power components from the advanced tillering stage and the booting stage impacted the user's and producer's accuracy, which is apparent in Figure 6(d). This effect is also observed for other decomposition techniques in Figure 6(a), Figure 6(b) and Figure 6(c), Consequently, misclassifications with other phenological stages were also evident (Figure 6(d) . This is indicated by the user's accuracy as shown in Table 4.

In contrast, the PA using the powers from the F3D, Y3D and An3D techniques for early and advanced tillering stages are $78.04 \%, 80.48 \%, 80.48 \%$; and $69.44 \%, 80.61 \%$, $91.67 \%$, respectively. As stated earlier, the confusion between the early tillering and bare field condition is marginal for An3D and Y3D due to comparable scattering power components. However for F3D, the confusion is higher between these two stages, as well as between the advanced tillering and maturity stages. This could be due to the high value of volume scattering computed by the F3D technique. For all these three model-based decomposition techniques, the average reduction in UA and PA is around $2 \%$ and $7 \%$, respectively, as compared to bare field condition. It can be noted that the differences between the PA of $\mathrm{MF} 3 \mathrm{CF}$ and other techniques are marginal. This small difference might be due to the advancement of crop growth which could be partially accredited to the volume models assumed in those techniques. However, based on different volume scattering models in those decomposition techniques, the $\mathrm{PA}$ varies from one technique to another. For MF3CF, the $P_{\mathrm{v}}^{\mathrm{FP}}$ component is due to the depolarized component of the scattering wave. Hence, in this case, the power components of MF3CF are able to capture the scattering characteristics from distributed crop structures. As a consequence, the PA gets improved as compared to F3D, An3D and Y3D. 
An anomaly in the accuracy between MF3CF and An3D techniques is evident during the advanced tillering stages compared to the bare field and early tillering stages (Figure 6(b)). The PA and UA using the An3D technique are marginally higher than that of the proposed method. This anomaly might be due to the combination of $P_{\mathrm{d}}^{\mathrm{FP}}$, $P_{\mathrm{s}}^{\mathrm{FP}}$ and $P_{\mathrm{v}}^{\mathrm{FP}}$ scattering power components in a unique manner. During this transition period from an early tillering to the booting stage, the amount of polarized and depolarized power components generates uncertainty in the classification among early tillering, advanced tillering and booting stage using the proposed technique.

During the booting stage, the scattering signature from rice is majorly comparable to the advanced tillering stage as the crop attains a semi-rigid structure. Hence, the PA using MF3CF is $82.06 \%$, while the UA is $86.48 \%$. On the other hand, the PA for F3D, Y3D and An3D are $69.23 \%, 79.92 \%$ and $82.06 \%$, while the UA is $71.04 \%, 76.92 \%$ and $82.06 \%$ respectively. It may be noted that during this period, the PA and UA for MF3CF and the F3D, Y3D, and An3D techniques are comparable, which could be due to similar scattering power components during this period. In particular, F3D, Y3D and An3D misclassify most of the booting stage crops as being in an advanced tillering stage because of the high volume scattering power computed by these methods during these two stages.

[Figure 7 about here.]

At the flowering stage, both PA and UA have increased as opposed to the booting stage. The PA and UA at this stage are $95.46 \%$ and $95.47 \%$ respectively using the MF3CF technique. In contrast, the PA and UA using the An3D method are $90.90 \%$ and $93.02 \%$, using the F3D are $81.81 \%$ and $87.80 \%$, and using the Y3D are $88.63 \%$ and $90.69 \%$, respectively. This increase in the accuracies might be due to the devel- 
opment of the anther in the rice. Most of the time, the flowering stage consists of heading and flowering stages as flowering begins after a day heading stage completed. Therefore, the fully visible panicle as well as the fully open flower which are ready to shed pollen on each other for pollination makes this separable from booting and other phenological stages.

During the maturity stage, both UA and PA records $100 \%$ using MF3CF technique. These accuracies at the maturity stage essentially indicate that the RF classifier has had no confusion with other phenology stages. However, confusion of the maturity stage with other advanced phenology stages is evident in Figure 6(a), Figure 6(b) and Figure 6(c). Hence, a lower estimate of PA and UA are apparent during this stage. These lower values might to due to the similarity in volume scattering components during these stages.

Therefore, the above classification accuracy in terms of PA and UA states that MF3CF decomposition parameters outperform the F3D, Y3D and An3D methods for rice phenology classification. We also observe this difference in the accuracies in terms of the overall accuracy and the kappa coefficient $(\kappa)$.

The overall accuracy using MF3CF is $91.17 \%$, while the overall accuracy using the F3D, Y3D and An3D methods are $76.89 \%, 84.03 \%$ and $82.77 \%$, respectively. Besides, $\kappa$ using the MF3CF is 0.91 , whereas, it is 0.82 for the $\mathrm{An} 3 \mathrm{D}, 0.73$ for the F3D and 0.81 for the Y3D methods. Although the $p$-value for both methodologies affirms the rejection of the null hypothesis, the $p$-value of MF3CF is much smaller than An3D, F3D and Y3D. An analysis-ready temporal phenological map of rice is shown in Figure 7. Therefore, the high $\kappa$ and the $p$-value in Table 5 suggests that classification using MF3CF holds excellent promise. 


\subsection{Dual co-polarimetric SAR data}

This section utilizes the obtained target characterization parameters from the proposed MF3CD decomposition technique to classify rice phenological stages. To evaluate the performance of these scattering power components for rice phenology classification, we extracted the DP data from Radarsat-2 FP SAR data. The variations of $\theta_{\mathrm{DP}}$ across all phenological stages of rice are shown in Figure 8 . The variations of $\theta_{\mathrm{DP}}, m_{\mathrm{DP}}$ and MF3CD scattering power components, were considered for the same four rice fields, as used for the analysis of the FP data.

[Figure 8 about here.]

At the initial stages of crop development (i.e., early leaf development), scattering from the soil usually dominates. Hence, $\theta_{\mathrm{DP}}$ is $\approx 30^{\circ}$ to $42^{\circ}$. The high values of $\theta_{\mathrm{DP}}$ are due to the presence of co-polarized correlations with the absence of the cross-polarized component.

As discussed in the earlier section, some of the fields had a small amount of surfaces roughness. The overall $\theta_{\mathrm{DP}}$ varies from $18^{\circ}$ to $42^{\circ}$. However, due to the presence of moderately smooth to smooth soil surface in most of the fields, the percentage of polarized scattering power components has greatly increased.

In particular, the interaction of EM waves with soil is dominated by the singlebounce scattering type, and therefore, the surface scattering power increases during this period. $m_{\mathrm{DP}}$ ranges between 0.6 to 0.8 , and the proportion of $P_{\mathrm{s}}^{\mathrm{DP}}$ power ranges between $60 \%$ to $80 \%$.

However, the $P_{\mathrm{v}}^{\mathrm{DP}}$ power varies from field to field depending on the surface roughness. For example, $\approx 40 \%$ of the diffused power component is apparent in a few fields where the soil surface condition was somewhat rough. 
Tillering started on 24 July 2019, but the crop density was very low at this period, and hence, the scattering from the soil surface was still dominant. However, crop emergence has slightly increased scattering randomness. Consequently, the values of $\theta_{\text {DP }}$ has decreased compared to 06 June 2019. During this period, it varies from $15^{\circ}$ to $30^{\circ}$.

Due to this increase in randomness, the values of $m_{\mathrm{DP}}$ has also decreased, and it ranges from 0.5 to 0.6 . These low values of $m_{\mathrm{DP}}$ influenced the scattering power components significantly. During this period, the proportion of $P_{\mathrm{s}}^{\mathrm{DP}}$ is similar to $P_{\mathrm{v}}^{\mathrm{DP}}$. Besides, the amount of $P_{\mathrm{d}}^{\mathrm{DP}}$ power has also increased due to the interaction of the EM wave with the stem-water configuration.

[Figure 9 about here.]

At the advanced tillering stage on $17 \mathrm{Aug}, \theta_{\mathrm{DP}}$ reveals a significant contribution of the even-bounce scattering mechanism. As stated earlier, this increase in the evenbounce scattering is due to the wave interaction among the rice stem and surface water.

Even-bounce scattering mechanism is apparent throughout the flowering stage. Besides, $m_{\text {DP }}$ values decreased from 24 July 2019 to 17 August 2019. However, throughout the phenology cycle, the cross-polarization component increases. Therefore, the absence of this cross-polarization component has lowered the values of $m_{\mathrm{DP}}$, in comparison to $m_{\mathrm{FP}}$. The values of $\theta_{\mathrm{DP}}$ and $m_{\mathrm{DP}}$ during these periods are $-5^{\circ}$ to $30^{\circ}$ and 0.2 to 0.6 .

Starting from the advanced tillering stage, $P_{\mathrm{v}}^{\text {DP }}$ powers prevails dominant. On 10 Sep, rice advanced to the booting stage. During this stage, values of $m_{\mathrm{DP}}$ have increased compared to 17 August 2019, which might be due to the presence of a weak 
cross-polarization component.

As a result, the magnitude of $P_{\mathrm{d}}^{\mathrm{DP}}$ powers has increased as compared to the previous date. A similar situation exists during the flowering stage on 04 Oct except for field F2. During this time, $P_{\mathrm{v}}^{\mathrm{DP}}$ and $P_{\mathrm{d}}^{\mathrm{DP}}$ powers coexists with significant proportion. For field $\mathrm{F} 2$, the $P_{\mathrm{v}}^{\mathrm{DP}}$ power is considerably higher than other components, which is due to high depolarization of the backscattered waves from this field.

During the maturity stage, multiple scattering from the canopy and soil is more apparent. Hence, on 21 November 2019, $\theta_{\text {DP }}$ fluctuates from $-8^{\circ}$ to $10^{\circ}$. The multiple scattering from the fields has increased the amount of depolarization in the backscattered EM wave. $m_{\mathrm{DP}}$ varies in the range of 0.2 to 0.4 . As a result, the magnitude of $P_{\mathrm{v}}^{\mathrm{DP}}$ is very high in each field. The values of $P_{\mathrm{v}}^{\mathrm{DP}}$ ranges from $60 \%$ to $80 \%$. Conversely, the values of $P_{\mathrm{s}}^{\mathrm{DP}}$ and $P_{\mathrm{d}}^{\mathrm{DP}}$ are significantly lower than $20 \%$.

[Table 6 about here.]

[Table 7 about here.]

[Figure 10 about here.]

Here we have shown rice phenology classification using the MF3CD scattering powers: $P_{\mathrm{d}}^{\mathrm{DP}}, P_{\mathrm{s}}^{\mathrm{DP}}$ and $P_{\mathrm{v}}^{\mathrm{DP}}$ from dual co-polarimetric SAR data. Additionally, the phenology classification accuracy is compared with the results obtained from FP SAR data. The producer's and user's accuracies are provided in Table 6 along with the global measurements in Table 7. We have also compared the proposed MF3CD technique's classification accuracy with the existing decomposition technique for DP SAR data proposed by Ullmann, Schmitt, and Jagdhuber (2016) (U2D). Additionally, the elements of the $\mathbf{T}_{2}$ coherency matrix (i.e. $T_{11}, T_{22}$ and $\left|T_{12}\right|$ ) are also used for phenology classification. 
It can be noted from Table 6 , that the PA and UA for bare field condition (BF) are identical at $95.83 \%$, which is similar to the classification accuracies using MF3CF for FP data. However, the PA of MF3CD has decreased by $\approx 5 \%$, which might be due to the changes in the degree of polarization because of the absence of cross-polarized component in the coherency elements. On the other hand, the PA for U2D and $\mathbf{T}_{2}$ matrix elements are $87.80 \%$ and $82.92 \%$, respectively, while the UAs are $97.29 \%$ and $97.14 \%$, respectively. Hence, high UA essentially indicates that some pixels from other phenological stages are classified as the BF stage. From the confusion matrix shown in Figure 10(b) and Figure 10(a) we can observe a mixing among early transplanted (ET) and BF stages. At this stage, this mixing might have increased the UA for these two techniques.

The PA and UA during the early tillering stage are $80.48 \%$ and $91.67 \%$, respectively, which could be due to the increase in the structural complexity of the crop. Besides, we describe the low accuracy of the early tillering stage by addressing the relative similarity in the values of $P_{\mathrm{s}}^{\mathrm{DP}}$ and $P_{\mathrm{v}}^{\mathrm{DP}}$ for the bare field, advanced tillering and maturity stages. The difference between the accuracies of MF3CD and MF3CF are marginal, which is due to similar depolarization structure and co-pol response. At this stage, the difference between $\mathrm{UA}$ of $\mathrm{MF} 3 \mathrm{CF}$ and $\mathrm{MF} 3 \mathrm{CD}$ is $\approx 2 \%$ and $\mathrm{PA}$ is $\approx 3 \%$. We also observe a similar decreasing trend in the accuracies for U2D and $\mathbf{T}_{2}$. The PA and UA of U2D are $84.62 \%$ and $80.49 \%$ and PA and UA of $\mathbf{T}_{2}$ are $79.48 \%$ and $75.61 \%$, respectively. At the advanced tillering, booting, and maturity stages, a similar pattern in the scattered power components produces significant confusion among the stages (Figure 10(c)], thereby decreasing PA and UA. Besides, an exception in the accuracies using MF3CF and MF3CD is evident during the booting period. The UA and PA using MF3CD technique are marginally higher than the MF3CF technique. 
However, the PA and UA for U2D and $\mathbf{T}_{2}$ are significantly lower during booting. This difference might be due to inadequate information about the scattering from a target acquired by DP SAR data.

[Figure 11 about here.]

During booting, the similar diffused power component creates confusion with the advanced tillering stage. Besides, being short duration booting and flowering stages arise alike polarimetric features in the co-pol response. However, coherence between the co-polar channels still exhibits a unique pattern compared to other phenological stages. This pattern might impact the scattering power components of the dual copolarimetric data, which depends only on the co-polar coherence term in the absence of the cross-polar component. On the other hand, as discussed earlier, the flowering stage shows a significant difference due to anther and fully visible heads. The presence of these crop morphological features generates a difference in the scattering mechanism, which increases the UA and PA at the flowering stage. The PA at the flowering stage is $90.90 \%$, while UA is $93.02 \%$. Furthermore, ample diffused scattering power component created considerable confusion during rice phenology classification in later phenological stages. Similar increase is also evident for T2 elements and U2D decomposition technique. The PA of T2 elements and U2D are $82.06 \%$ and $82.04 \%$, respectively, while UAs are $91.43 \%$ and $91.43 \%$, respectively.

However, at the maturity stage, although the $P_{\mathrm{v}}^{\mathrm{DP}}$ power is dominant, and the pattern of $P_{\mathrm{d}}^{\mathrm{DP}}$ and $P_{\mathrm{s}}^{\mathrm{DP}}$ powers might have produced high PA $(100 \%)$ and UA $(70.60 \%)$. In contrast, the absence of volume or diffused component in $\mathrm{U} 2 \mathrm{D}$ and $\mathbf{T}_{2}$ elements has lowered the PA and UA. The PA and UA for $\mathbf{T}_{2}$ elements are $64.86 \%$ and $46.16 \%$, respectively. The overall accuracy using MF3CD is $86.97 \%$ with a $\kappa$ of 0.84 (Table 7 ). 
On the other hand, the overall classification accuracy using U2D and $\mathbf{T}_{2}$ elements are $76.47 \%$ and $71.00 \%$, respectively. The analysis-ready map of rice phenology using MF3CD is shown in Figure 11. Therefore, it can be observed that the overall classification accuracy using our proposed MF3CD decomposition technique is comparable to FP data. These overall accuracy results infer the potential of employing our MF3CD decomposed scattering power components for rice phenology classification.

\section{Conclusions}

This study proposed a model-free scattering power decomposition framework for fullpolarimetric (FP), and dual-polarimetric (DP) SAR data using the Kennaugh matrix elements $k_{i j}: i, j=1,2,3,4$. We used the $k_{11}$ and $k_{44}$ elements of the Kennaugh matrix and the $n \mathrm{D}$ Barakat degree of polarization to describe the scattering-type parameter. We then used the scattering-type parameter as a geometrical factor to compute the three scattering power components individually for full (MF3CF) and for dual co-pol (MF3CD) from the total scattered power. We utilized the full-pol (FP) and the extracted dual co-pol (DP) data from the C-band Radarsat-2 SAR data. The images were acquired through the entire expanse of rice development. The scattering power components vary with changes in rice phenology stages. The potential of these scattering power components was also assessed for characterizing rice growth condition.

The analysis of the results shows that the proposed scattering power components are sensitive to rice phenology and hence useful to classify different rice phenology stages. The power components can discriminate rice growth stages due to scattering purity changes based on the development of unique canopy structures as the season progresses. The overall classification accuracy using the proposed power components for the FP SAR data is better than the An and Yang three-component, Freeman and 
Durden three-component, and Yamaguchi three-component decomposition techniques.

The overall accuracy using the proposed power components for the DP SAR data is better than using only the matrix elements or the Ullmann two-component decomposition technique. Moreover, the overall accuracy (OA) of using the proposed power components for the DP SAR data is only marginally lower than the FP SAR data. Like the FP SAR data, this study demonstrates the potential of DP SAR data for efficient rice phenology classification. In this study the OA using MF3CF and MF3CD are $91.17 \%$ and $86.97 \%$, respectively.

Even though the results are quite encouraging, the study has some limitations based on the available data sets. On the one hand, a denser time series of SAR data might have reduced confusion between certain phenological stages, particularly booting and flowering. In addition to this, enhancing the classification algorithm might also improve the mapping of intermediate phenological stages. Further, dense temporal data throughout the season can assist better cultivation practices. Besides this, short revisit times are useful to monitor phenology information closely.

On the other hand, the proposed decomposition technique is model-free. Therefore, the scattering power components are unique and unambiguous. Within the decomposition framework, the target characterization parameters and the scattering power components are roll-invariant. However, the proposed technique has a limitation based on the nature of the coherency matrix for dual co-pol SAR data. It is apparent that the framework can not be extended to obtain the target asymmetry component either in terms of the scattering power components or the target characterization parameters. Nevertheless, all the scattering power components of MF3CF and MF3CD are nonnegative and stable (i.e., the scattering power components does not change abruptly with a small perturbation of the coherency matrix). 
Please note that in this work, the studies were conducted in a semi-arid region.

However, the cultivation and management practice of rice could differ around the globe. Therefore, one must expand this method for other sites and other types of crops. Future studies should also include radar images acquired at X- and L-band, such as the TerraSAR-X and ALOS-2 satellites. At high frequencies, X-band could discriminate the initial growth stages from advanced growth stages. However, at lower frequencies, we expect less sensitivity to the initial emergence of the plants, and, as such, L-band might be useful to discriminate more advanced phenology stages of crops. These multi-frequency analyses would lead to a better understanding of crop phenology to the farming user community.

\section{Reproducibility and Replicability}

Following the guidelines presented in Frery, Gomez, and Medeiros (2020), we made available the code that supports the reproducibility and replicability of this work in a Github repository: https://github.com/Subho07/MF3CD.

\section{Acknowledgements}

This work was supported in part by the Spanish Ministry of Science, Innovation and Universities, the State Agency of Research (AEI), and the European Funds for Regional Development (EFRD) under Project TEC2017-85244-C2-1-P. The authors would like to thank the Canadian Space Agency and MAXAR Technologies Ltd. (formerly MDA) for providing Radarsat-2 images through the Joint Experiment for Crop Assessment and Monitoring (JECAM) SAR Inter-comparison Experiment network. The authors are also thankful to Andhra Pradesh Space Application Centre (AP- 
SAC), ITE \& C Department, Government of Andhra Pradesh for their support during field campaigns.

\section{Disclosure statement}

No potential conflict of interest was reported by the authors.

\section{References}

Adhikari, B, MK Bag, MK Bhowmick, and C Kundu. 2011. "Status paper on rice in West Bengal." Rice Knowledge Management Portal http://www.rkmp.co.in

An, W., Y. Cui, and J. Yang. 2010. "Three-Component Model-Based Decomposition for Polarimetric SAR Data." IEEE Transactions on Geoscience and Remote Sensing 48 (6): 2732 2739.

Barakat, Richard. 1977. "Degree of polarization and the principal idempotents of the coherency matrix." Optics Communications 23 (2): 147-150.

Born, Max, and Emil Wolf. 2013. Principles of optics: electromagnetic theory of propagation, interference and diffraction of light. Elsevier.

Bouvet, Alexandre, Thuy Le Toan, and Nguyen Lam-Dao. 2009. "Monitoring of the rice cropping system in the Mekong Delta using ENVISAT/ASAR dual polarization data." IEEE Transactions on Geoscience and Remote Sensing 47 (2): 517-526.

Cloude, S. 1986. "Group theory and polarisation algebra." Optik 75: 26-36.

Cloude, Shane R, and Eric Pottier. 1997. "An entropy based classification scheme for land applications of polarimetric SAR." IEEE Transactions on Geoscience and Remote Sensing 35 (1): $68-78$.

Corcione, V, F Nunziata, L Mascolo, and M Migliaccio. 2016. "A study of the use of COSMOSkyMed SAR PingPong polarimetric mode for rice growth monitoring." International Journal of Remote Sensing 37 (3): 633-647. 
De Bernardis, Caleb G, Fernando Vicente-Guijalba, Tomas Martinez-Marin, and Juan M Lopez-Sanchez. 2015. "Estimation of key dates and stages in rice crops using dualpolarization SAR time series and a particle filtering approach." IEEE Journal of Selected Topics in Applied Earth Observations and Remote Sensing 8 (3): 1008-1018.

Dey, S., A. Bhattacharya, D. Ratha, D. Mandal, and A. C. Frery. 2020. "Target Characterization and Scattering Power Decomposition for Full and Compact Polarimetric SAR Data." IEEE Transactions on Geoscience and Remote Sensing 1-18.

Dey, Subhadip, Avik Bhattacharya, Debanshu Ratha, Dipankar Mandal, Heather McNairn, Juan M. Lopez-Sanchez, and Y.S. Rao. 2020a. "Novel clustering schemes for full and compact polarimetric SAR data: An application for rice phenology characterization." ISPRS Journal of Photogrammetry and Remote Sensing 169: 135 - 151.

Dey, Subhadip, Dipankar Mandal, Laura Dingle Robertson, Biplab Banerjee, Vineet Kumar, Heather McNairn, Avik Bhattacharya, and YS Rao. 2020b. "In-season crop classification using elements of the Kennaugh matrix derived from polarimetric RADARSAT-2 SAR data." International Journal of Applied Earth Observation and Geoinformation 88: 102059.

Erten, Esra, Juan M Lopez-Sanchez, Onur Yuzugullu, and Irena Hajnsek. 2016. "Retrieval of agricultural crop height from space: A comparison of SAR techniques." Remote Sensing of Environment 187: 130-144.

Freeman, Anthony, and Stephen L Durden. 1998. "A three-component scattering model for polarimetric SAR data." IEEE Transactions on Geoscience and Remote Sensing 36 (3): 963-973.

Frery, A. C., L. Gomez, and A. C. Medeiros. 2020. "A Badging System for Reproducibility and Replicability in Remote Sensing Research." IEEE J. Sel. Topics Appl. Earth Observ. Remote Sens. 13: 4988-4995.

Huynen, J R. 1970. "Phenomenological theory of radar targets." PhD dissertation, Technical Univ., Delf, The Netherlands.

Koay, Jun-Yi, Chue-Poh Tan, Ka-Sing Lim, Saiful Bahari bin Abu Bakar, Hong-Tat Ewe, Hean- 
Teik Chuah, and Jin-Au Kong. 2007. "Paddy fields as electrically dense media: Theoretical modeling and measurement comparisons." IEEE Transactions on Geoscience and Remote Sensing 45 (9): 2837-2849.

Koppe, Wolfgang, Martin L Gnyp, Christoph Hütt, Yinkun Yao, Yuxin Miao, Xinping Chen, and Georg Bareth. 2013. "Rice monitoring with multi-temporal and dual-polarimetric TerraSAR-X data." International Journal of Applied Earth Observation and Geoinformation 21: $568-576$

Küçük, Çağlar, Gülşen Taşkın, and Esra Erten. 2016. "Paddy-rice phenology classification based on machine-learning methods using multitemporal co-polar X-band SAR images." IEEE Journal of Selected Topics in Applied Earth Observations and Remote Sensing 9 (6): $2509-2519$.

Kurosu, Takashi, Masaharu Fujita, and Kazuo Chiba. 1995. "Monitoring of rice crop growth from space using the ERS-1 C-band SAR." IEEE Transactions on Geoscience and Remote Sensing 33 (4): 1092-1096.

Lampayan, RM, JE Faronilo, TP Tuong, AJ Espiritu, JL De Dios, RS Bayot, CS Bueno, and Y Hosen. 2015. "Effects of seedbed management and delayed transplanting of rice seedlings on crop performance, grain yield, and water productivity." Field Crops Research 183: 303314 .

Lawrence, Rick L, Shana D Wood, and Roger L Sheley. 2006. "Mapping invasive plants using hyperspectral imagery and Breiman Cutler classifications (RandomForest)." Remote Sensing of Environment 100 (3): 356-362.

Le Toan, Thuy, Henri Laur, Eric Mougin, and Armando Lopes. 1989. "Multitemporal and dual-polarization observations of agricultural vegetation covers by X-band SAR images." IEEE Transactions on Geoscience and Remote Sensing 27 (6): 709-718.

Le Toan, Thuy, Florence Ribbes, Li-Fang Wang, Nicolas Floury, Kung-Hau Ding, Jin Au Kong, Masaharu Fujita, and Takashi Kurosu. 1997. "Rice crop mapping and monitoring using ERS1 data based on experiment and modeling results." IEEE Transactions on Geoscience and 
Remote Sensing 35 (1): 41-56.

Lee, Jong-Sen, and Eric Pottier. 2009. Polarimetric radar imaging: from basics to applications. CRC press.

Li, Kun, Brian Brisco, Shao Yun, and Ridha Touzi. 2012. "Polarimetric decomposition with RADARSAT-2 for rice mapping and monitoring." Canadian Journal of Remote Sensing 38 (2): 169-179.

Lopez-Sanchez, J. M., S. R. Cloude, and J. D. Ballester-Berman. 2012. "Rice Phenology Monitoring by Means of SAR Polarimetry at X-Band." IEEE Transactions on Geoscience and Remote Sensing 50 (7): 2695-2709.

Lopez-Sanchez, Juan M, J David Ballester-Berman, and Irena Hajnsek. 2012. "First results of rice monitoring practices in Spain by means of time series of TerraSAR-X dual-pol images." IEEE Journal of Selected Topics in applied earth observations and remote sensing 4 (2): $412-422$.

Lopez-Sanchez, Juan M, Fernando Vicente-Guijalba, J David Ballester-Berman, and Shane R Cloude. 2014. "Polarimetric response of rice fields at C-band: Analysis and phenology retrieval." IEEE Transactions on Geoscience and Remote Sensing 52 (5): 2977-2993.

Mahajan, Gulshan, TS Bharaj, and J Timsina. 2009. "Yield and water productivity of rice as affected by time of transplanting in Punjab, India." Agricultural Water Management 96 (3): $525-532$.

Mandal, D, V Kumar, YS Rao, A Bhattacharya, and KV Ramana. 2019. Experimental Field Campaigns at Vijayawada Test Site. Technical Report MRS2019TR02. Microwave Remote Sensing Lab, India. http://doi.org/10.17605/OSF.IO/DN3E8.

Moldenhauer, KEWC, and Nathan Slaton. 2001. "Rice growth and development." Rice production handbook 192: 7-14.

Réfrégier, Philippe, François Goudail, Pierre Chavel, and Ari Friberg. 2004. "Entropy of partially polarized light and application to statistical processing techniques." JOSA A 21 (11): $2124-2134$. 
Tian, Haifeng, Mingquan Wu, Li Wang, and Zheng Niu. 2018. "Mapping early, middle and late rice extent using Sentinel-1A and Landsat-8 data in the Poyang lake plain, China." Sensors $18(1): 185$.

Torbick, Nathan, Diya Chowdhury, William Salas, and Jiaguo Qi. 2017. "Monitoring rice agriculture across Myanmar using time series Sentinel- 1 assisted by Landsat- 8 and PALSAR2." Remote Sensing 9 (2): 119.

Touzi, Ridha. 2006. "Target scattering decomposition in terms of roll-invariant target parameters." IEEE Transactions on Geoscience and Remote Sensing 45 (1): 73-84.

Ullmann, Tobias, Andreas Schmitt, and Thomas Jagdhuber. 2016. "Two component decomposition of dual polarimetric HH/VV SAR data: Case study for the tundra environment of the Mackenzie Delta region, Canada." Remote Sensing 8 (12): 1027.

Yamaguchi, Y., A. Sato, W-M Boerner, R. Sato, and H. Yamada. 2011. "Four-Component Scattering Power Decomposition With Rotation of Coherency Matrix." IEEE Trans. Geosci. Remote Sens. 49 (6): 2251-2258.

Yang, Zhi, Yun Shao, Kun Li, Qingbo Liu, Long Liu, and Brian Brisco. 2017. "An improved scheme for rice phenology estimation based on time-series multispectral HJ-1A/B and polarimetric RADARSAT-2 data." Remote Sensing of Environment 195: 184-201.

Yuzugullu, Onur, Esra Erten, and Irena Hajnsek. 2015. "Rice growth monitoring by means of X-band co-polar SAR: Feature clustering and BBCH scale." IEEE Geoscience and Remote Sensing Letters 12 (6): 1218-1222.

Zhang, Yuan, Xiaohui Liu, Shiliang Su, and Cuizhen Wang. 2014. "Retrieving canopy height and density of paddy rice from Radarsat-2 images with a canopy scattering model." International Journal of Applied Earth Observation and Geoinformation 28: 170-180. 


\section{List of Figures}

1 Pauli RGB image acquired on 24 July, 2019 over the JECAM test site over Vijayawada, India. Region 1 and region 2 are the example subsets

of sampling locations within the study area. The yellow box indicates

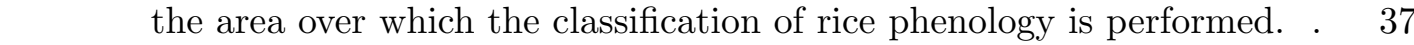

$2 \quad$ Crop morphological characteristics across phenological stages . . . . . 38

$3 \quad$ Variation of (a) $1+\sin 2 \theta_{n}$ and (b) $1-\sin 2 \theta_{n}$ for $\theta_{n} \in\left[-45^{\circ}, 45^{\circ}\right]$. . . 39

a 4 Variation in $\theta_{\mathrm{FP}}$ through the rice season with in the extent marked in yellow colour in Figure 1 . . . . . . . . . . . . . . . . . . . 40

5 Variation of $m_{\mathrm{FP}}, \theta_{\mathrm{FP}}$ and MF3CF scattering power components over the rice growing seasons . . . . . . . . . . . . . . . . . 41

6 The confusion matrices (in \%) among different phenological stages of rice (BF: Bare field, ET: Early tillering, AT: Advanced tillering, B: Booting, F: Flowering, M: Maturity). . . . . . . . . . . . . . 42

7 Variations in clusters using the MF3CF scattering power components for the rice growing season with in the extent marked in yellow colour in Figure 1 . . . . . . . . . . . . . . . . . . . . . 43

$8 \quad$ Variation in $\theta_{\text {DP }}$ through the rice season with in the extent marked in yellow colour in Figure 1 . . . . . . . . . . . . . . . . . . . . . 44

$9 \quad$ Variation of $m_{\mathrm{DP}}, \theta_{\mathrm{DP}}$ and MF3CD scattering power components over

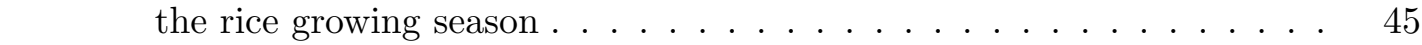

10 The confusion matrices (in \%) among different phenological stages

of rice (BF: Bare field, ET: Early tillering, AT: Advanced tillering,

B: Booting, F: Flowering, M: Maturity) $\ldots \ldots \ldots$. . . . . . . . 46 

rice growing season with in the extent marked in yellow colour in Figure 1147

\section{List of Tables}

$1 \quad$ Radarsat-2 data acquired for the Indian test site . . . . . . . . . . . 48

$2 \quad$ Description of rice morphology at different growth stages . . . . . . . 49

$3 \quad$ Statistics (mean \pm standard deviation) of bio-physical and soil param-

eters at different phenology stages of rice. Here, PH: plant height, PAI:

\begin{tabular}{|l|l|}
\hline plant area index, SM: soil moisture and NA: No measurements available 50 & 0
\end{tabular}

4 Producer's and User's accuracy of phenology stages of rice for MF3CF,

An3D, F3D and Y3D decomposed power components using a RF classi-

fier. BF: Bare field, ET: Early tillering, AT: Advanced tillering, B: Boot-

ing, F: Flowering, M: Maturity, PA: Producer's accuracy, UA: User's

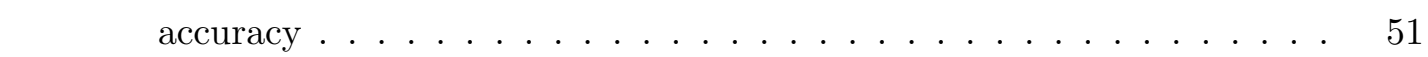

$5 \quad$ Global Measures for FP decomposition techniques $\ldots \ldots \ldots$. . . . . 52

6 Producer's and User's accuracy of phenology stages of rice for MF3CD,

U2D and $\mathbf{T}_{2}$ matrix elements using a RF classifier. BF: Bare field,

ET: Early tillering, AT: Advanced tillering, B: Booting, F: Flowering,

M: Maturity, PA: Producer's accuracy, UA: User's accuracy. The results

are separately compared with MF3CF. . . . . . . . . . . . 53

$7 \quad$ Global Measures for DP decomposition techniques . . . . . . . . . . 54 


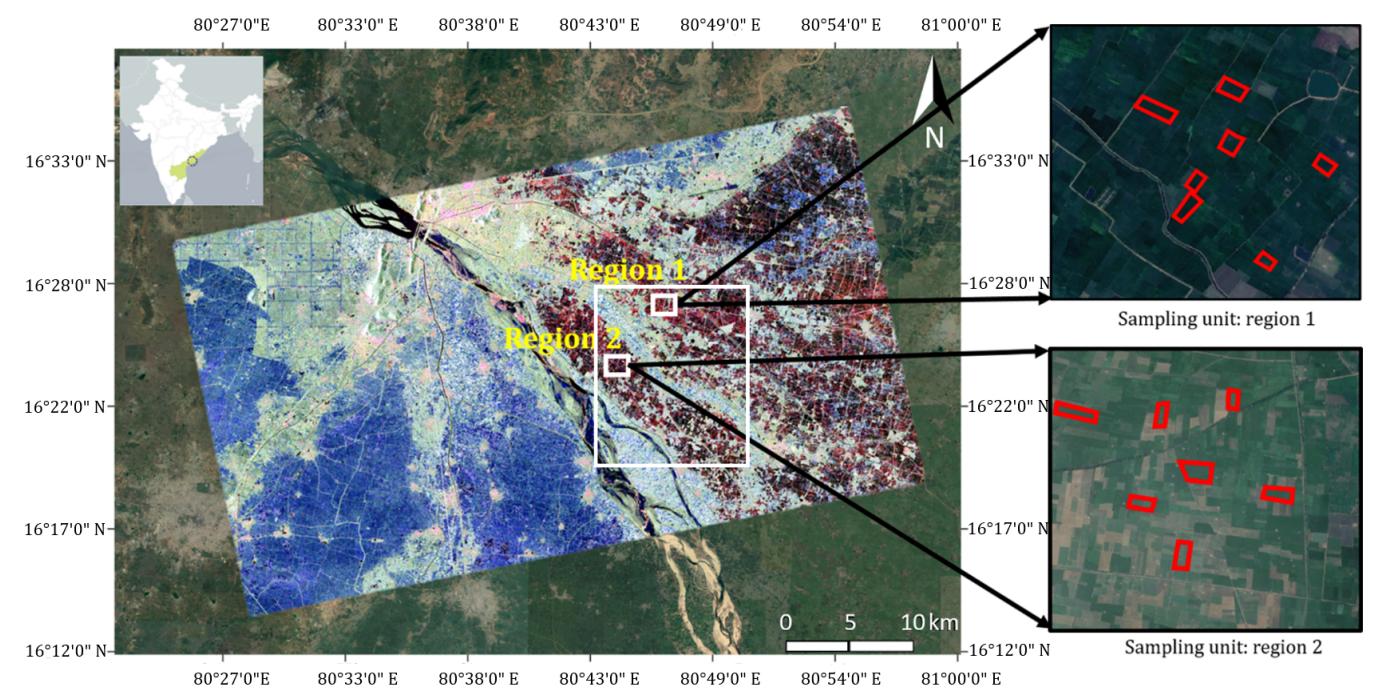

Figure 1. Pauli RGB image acquired on 24 July, 2019 over the JECAM test site over Vijayawada, India. Region 1 and region 2 are the example subsets of sampling locations within the study area. The yellow box indicates the area over which the classification of rice phenology is performed. 


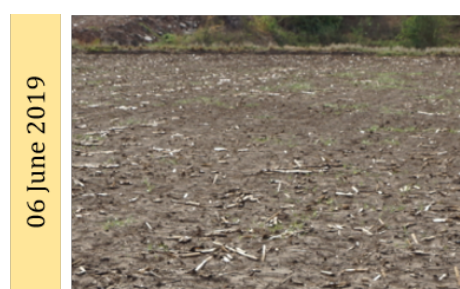

(a) Bare field

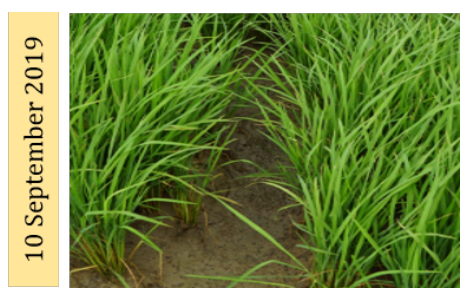

(d) Booting

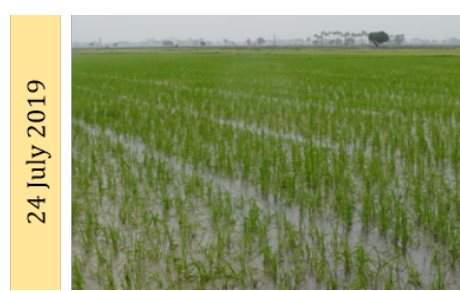

(b) Early tillering

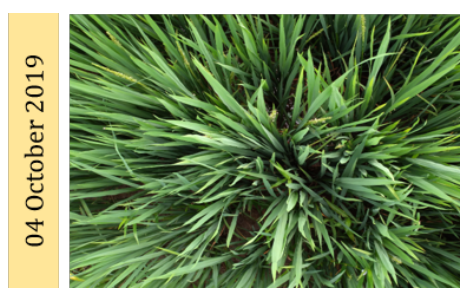

(e) Flowering

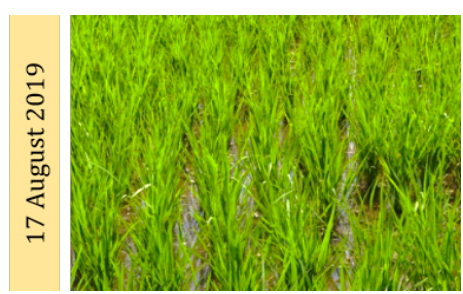

(c) Advanced tillering

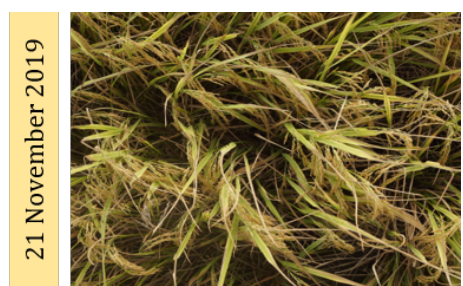

(f) Maturity

Figure 2. Crop morphological characteristics across phenological stages 


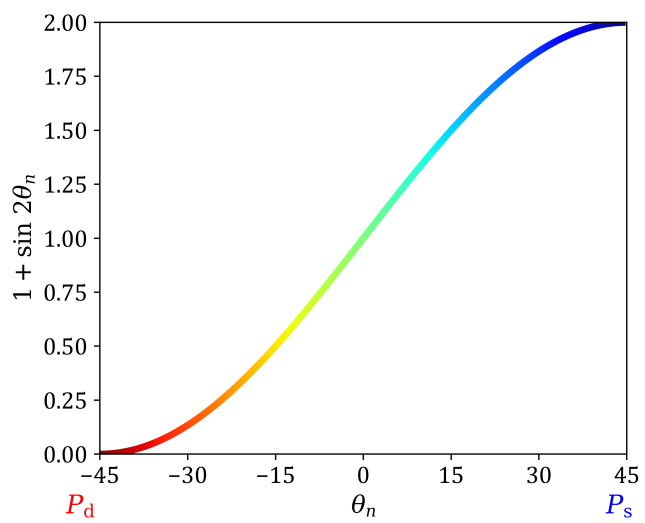

(a)

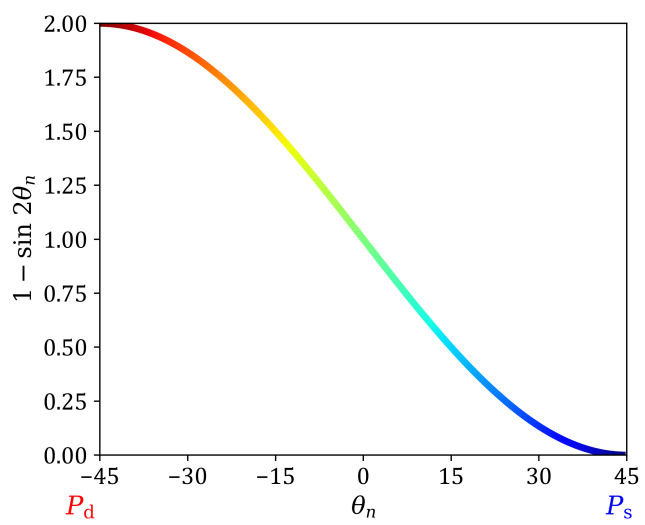

(b)

Figure 3. Variation of (a) $1+\sin 2 \theta_{n}$ and (b) $1-\sin 2 \theta_{n}$ for $\theta_{n} \in\left[-45^{\circ}, 45^{\circ}\right]$. 


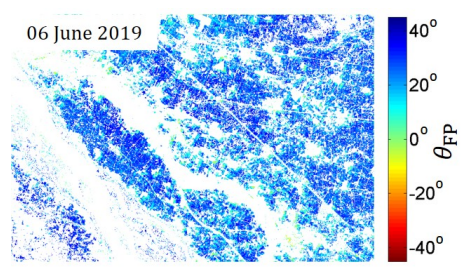

(a) 06 June 2019

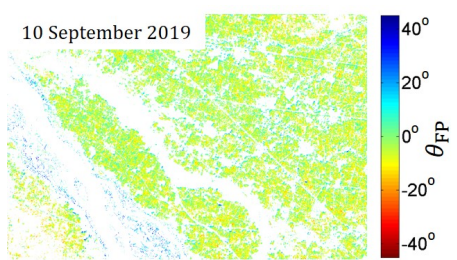

(d) 10 September 2019

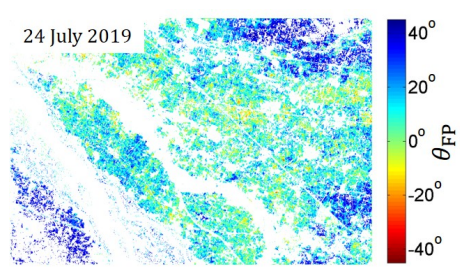

(b) 24 July 2019

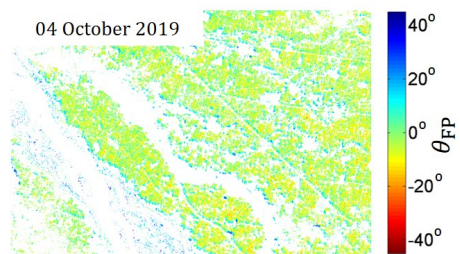

(e) 04 October 2019

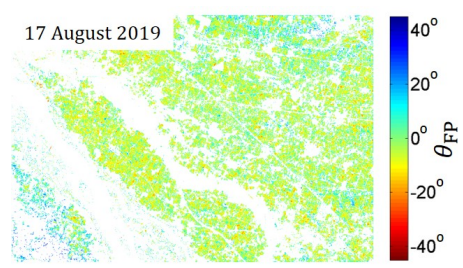

(c) 17 August 2019

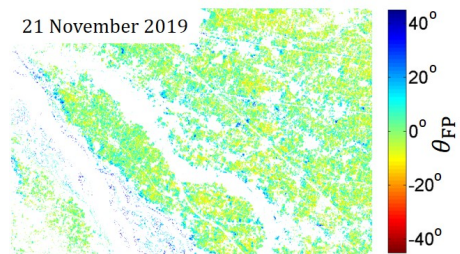

(f) 21 November 2019

Figure 4. Variation in $\theta_{\mathrm{FP}}$ through the rice season with in the extent marked in yellow colour in Figure 1 


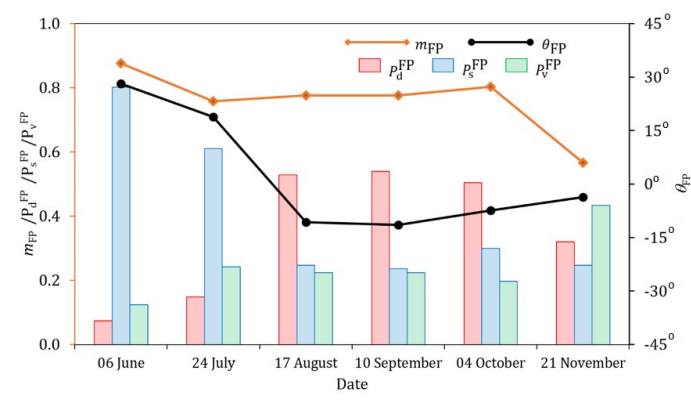

(a) F1

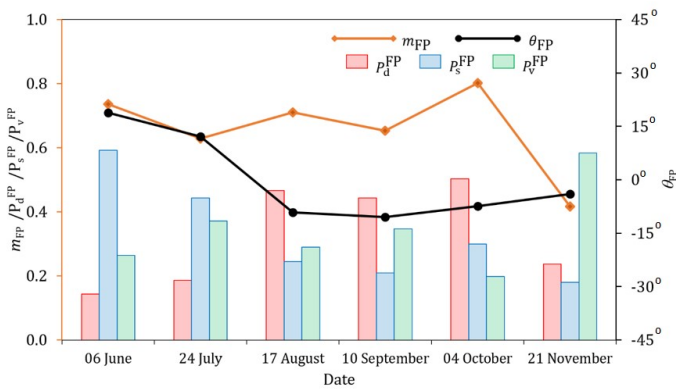

(c) F3

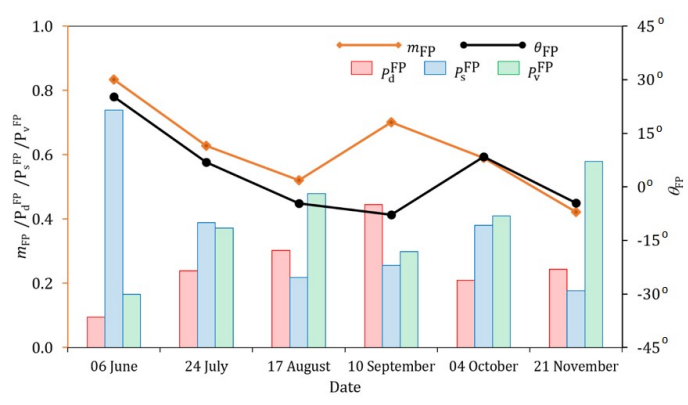

(b) F2

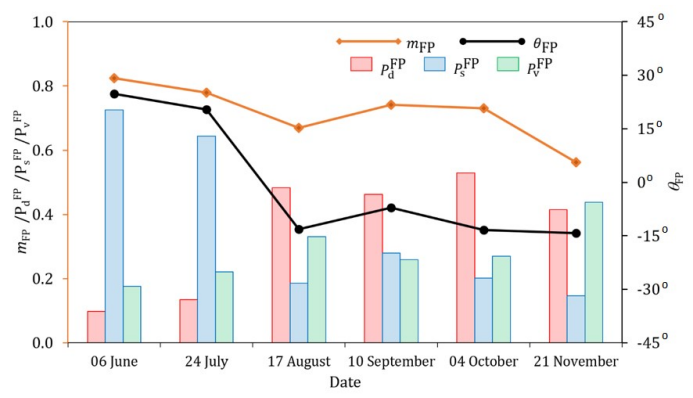

(d) F4

Figure 5. Variation of $m_{\mathrm{FP}}, \theta_{\mathrm{FP}}$ and $\mathrm{MF} 3 \mathrm{CF}$ scattering power components over the rice growing seasons 


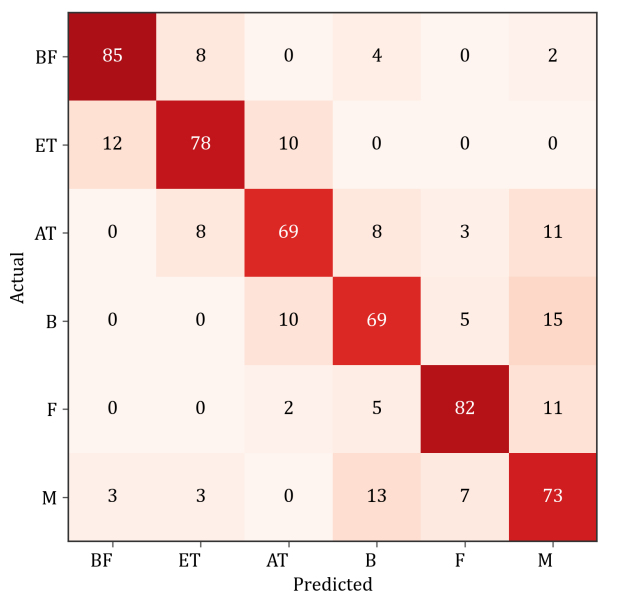

(a) F3D

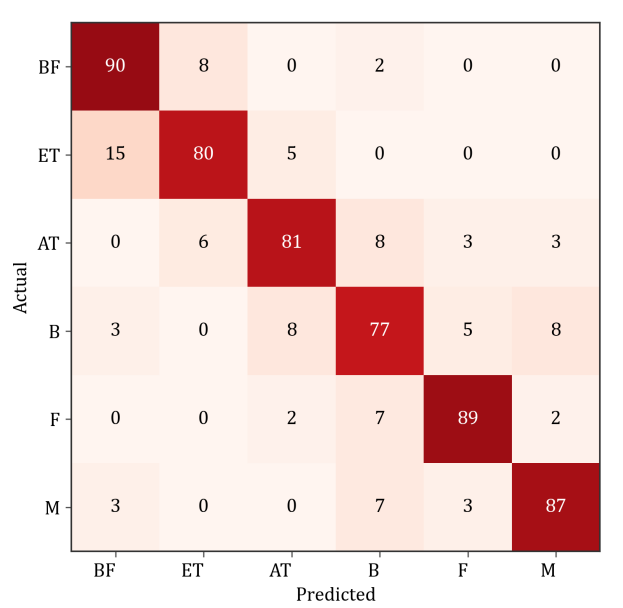

(c) Y3D
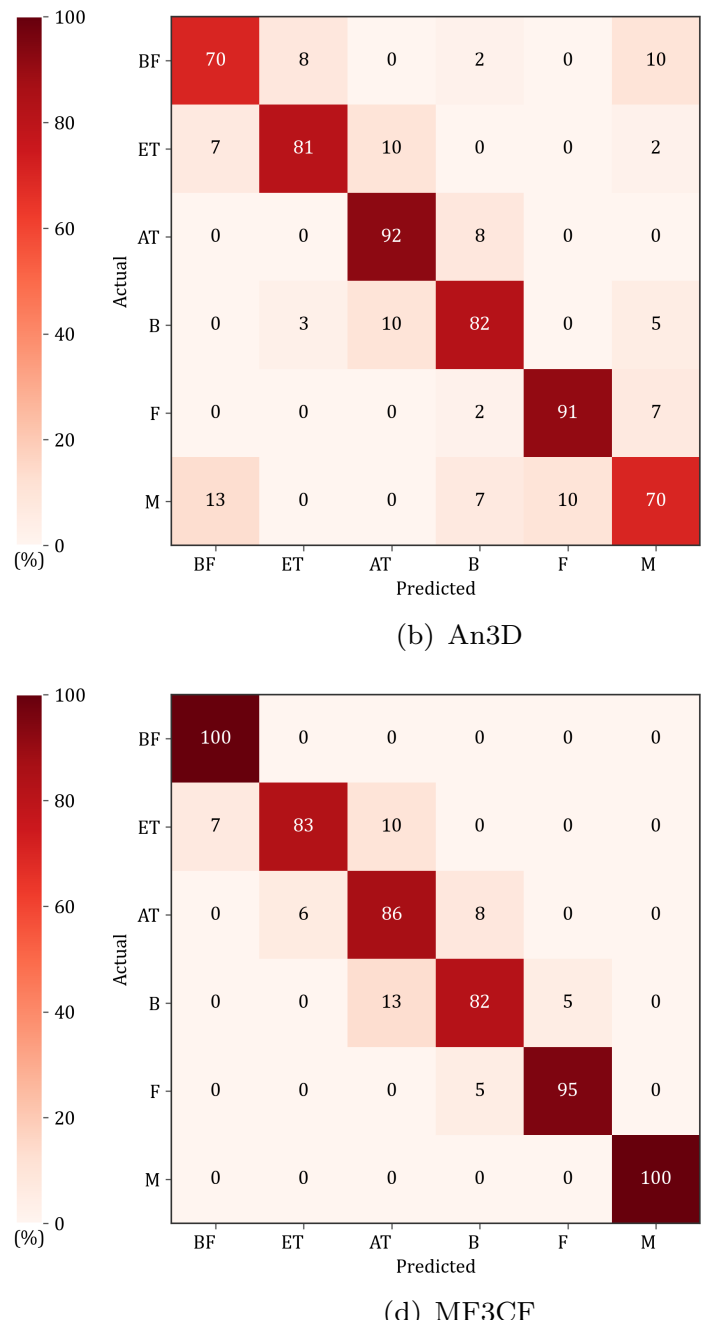

(b) An3D
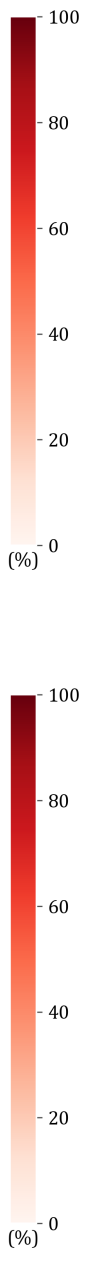

(d) $\mathrm{MF} 3 \mathrm{CF}$

Figure 6. The confusion matrices (in \%) among different phenological stages of rice (BF: Bare field, ET: Early tillering, AT: Advanced tillering, B: Booting, F: Flowering, M: Maturity). 


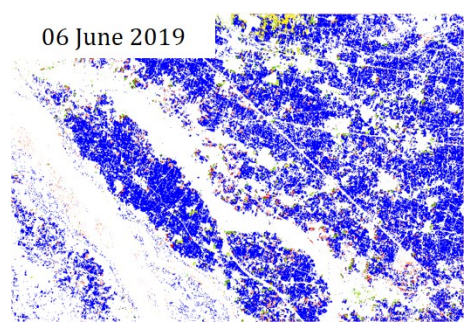

(a) 06 June

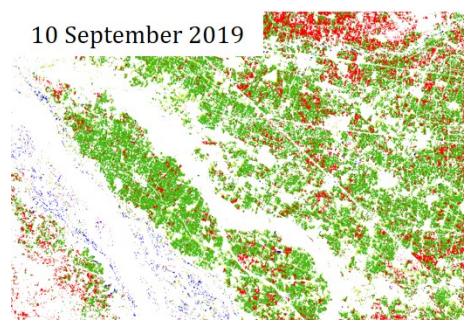

(d) 10 September 2019

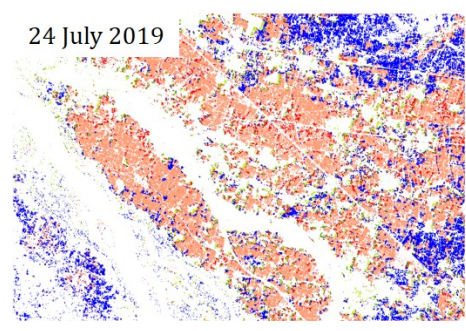

(b) 24 July 2019

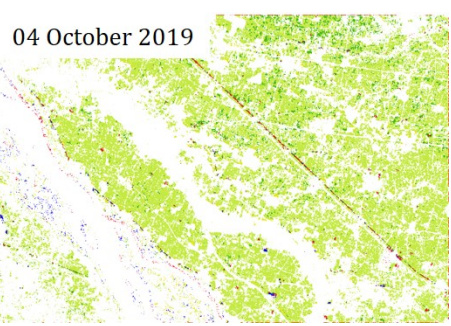

(e) 04 October 2019

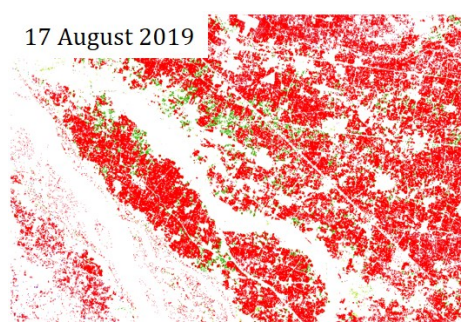

(c) 17 August 2019

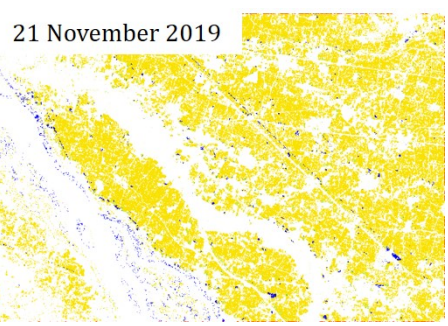

(f) 21 November 2019

Bare field Early tillering

Figure 7. Variations in clusters using the MF3CF scattering power components for the rice growing season with in the extent marked in yellow colour in Figure 1 


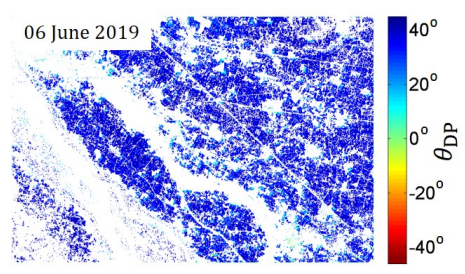

(a) 06 June 2019

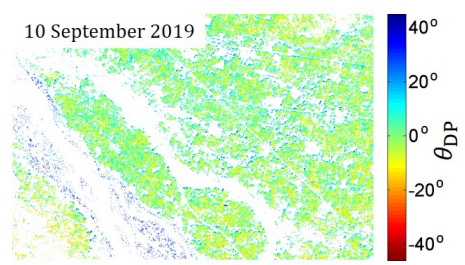

(d) 10 September 2019

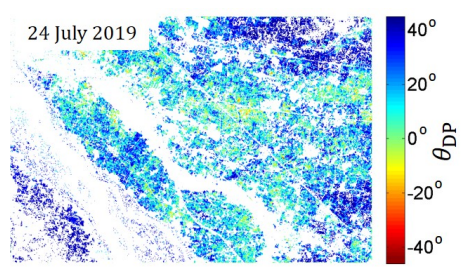

(b) 24 July 2019

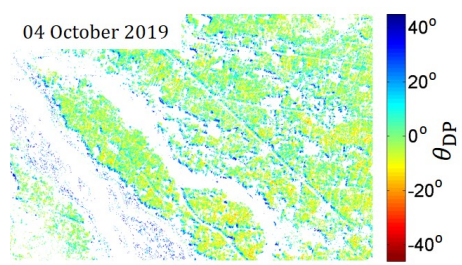

(e) 04 October 2019

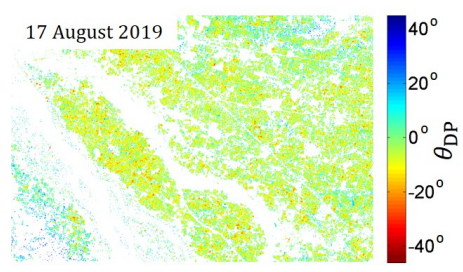

(c) 17 August 2019

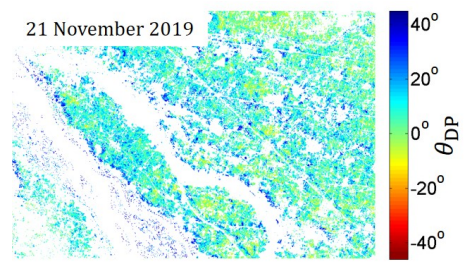

(f) 21 November 2019

Figure 8. Variation in $\theta_{\mathrm{DP}}$ through the rice season with in the extent marked in yellow colour in Figure 1 


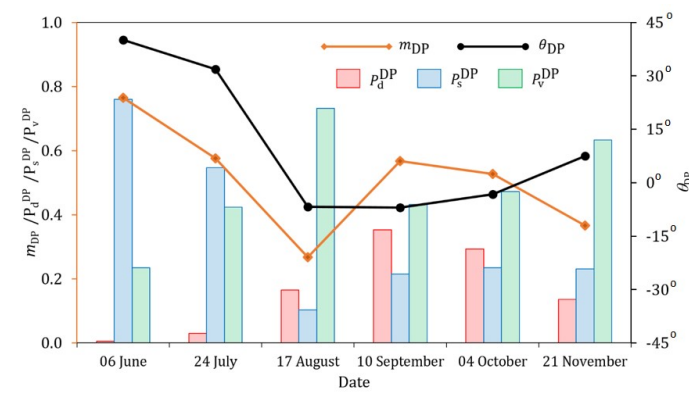

(a) F1

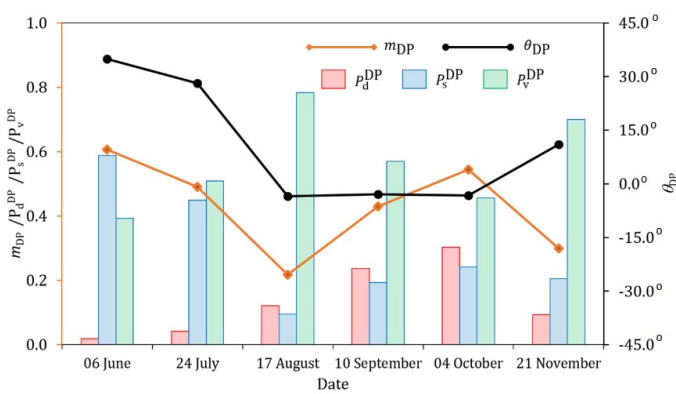

(c) F3

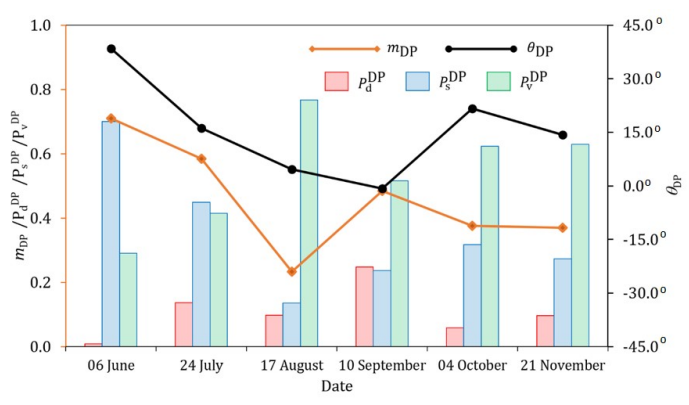

(b) F2

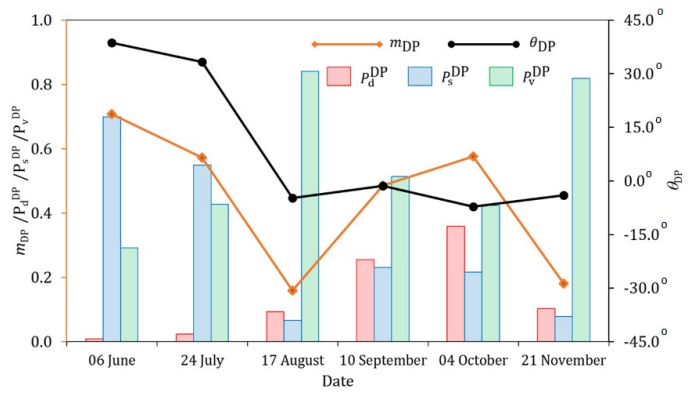

(d) F4

Figure 9. Variation of $m_{\mathrm{DP}}, \theta_{\mathrm{DP}}$ and MF3CD scattering power components over the rice growing season 


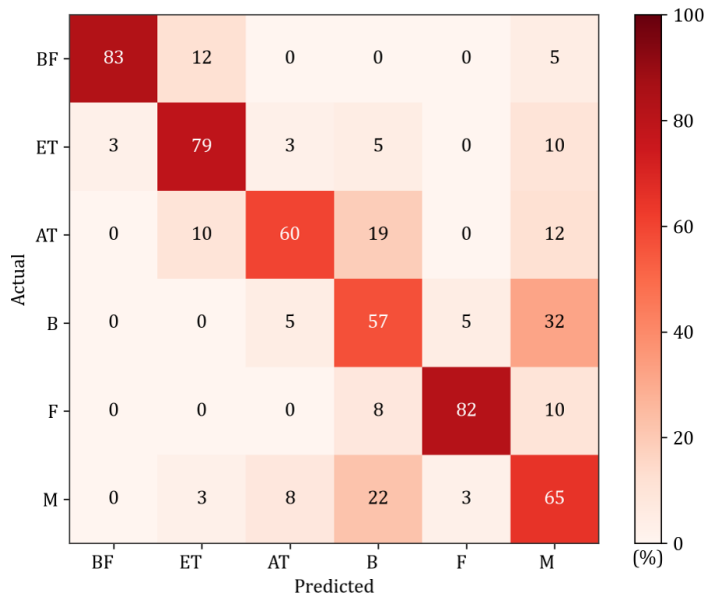

(a) $\mathbf{T}_{2}$ Elements

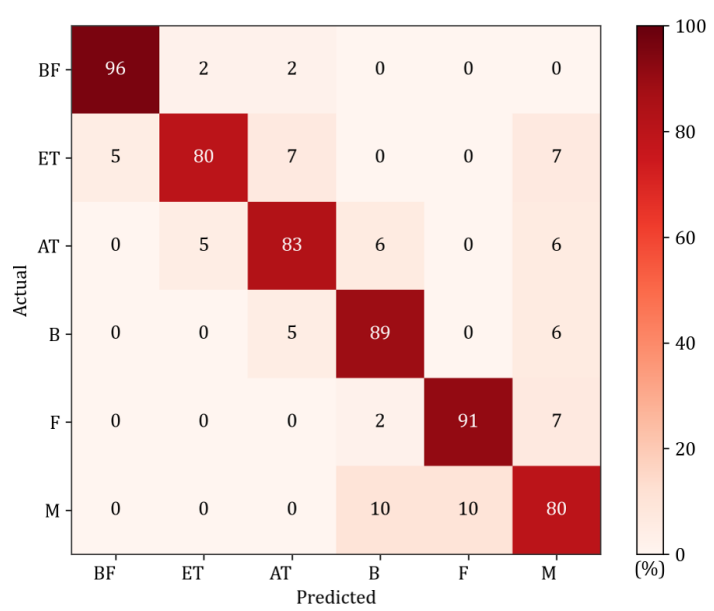

(c) $\mathrm{MF} 3 \mathrm{CD}$

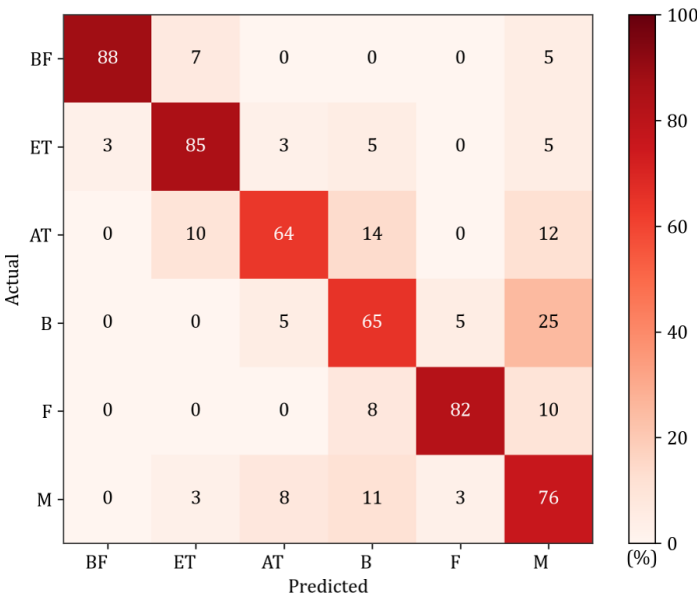

(b) U2D

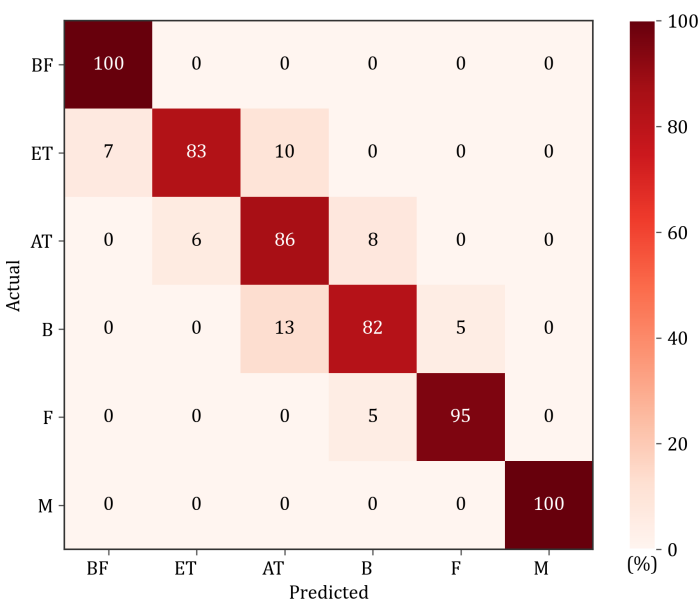

(d) $\mathrm{MF} 3 \mathrm{CF}$

Figure 10. The confusion matrices (in \%) among different phenological stages of rice (BF: Bare field, ET: Early tillering, AT: Advanced tillering, B: Booting, F: Flowering, M: Maturity) 


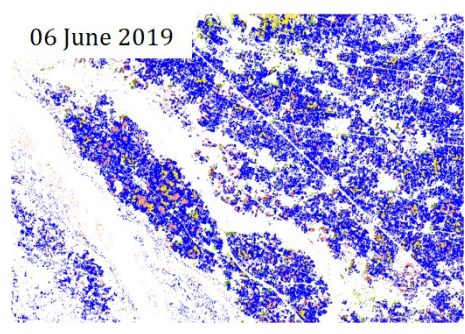

(a) 06 June 2019

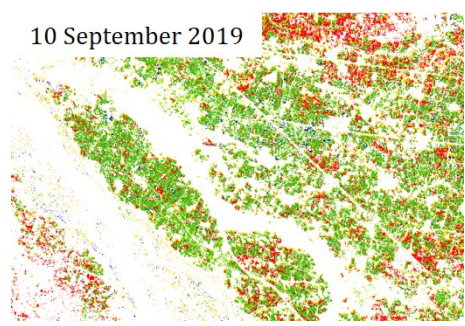

(d) 10 September 2019

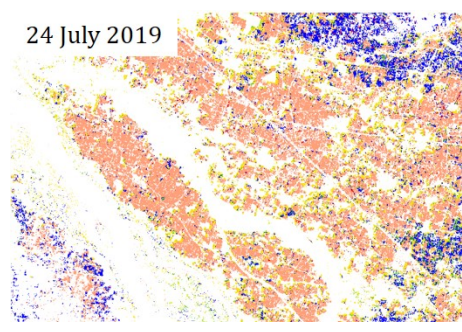

(b) 24 July 2019

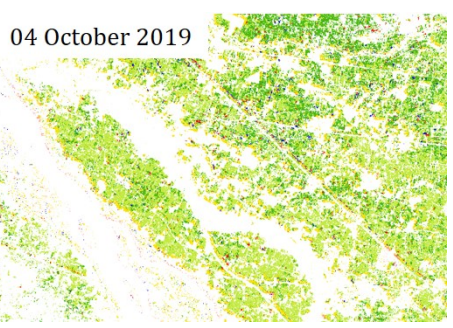

(e) 04 October 2019

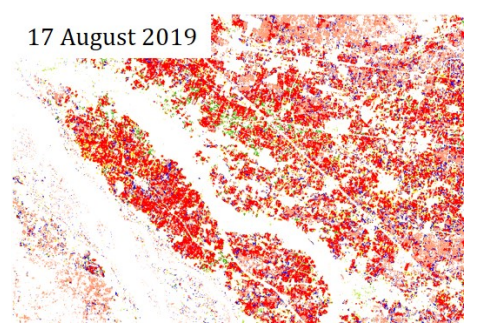

(c) 17 August 2019

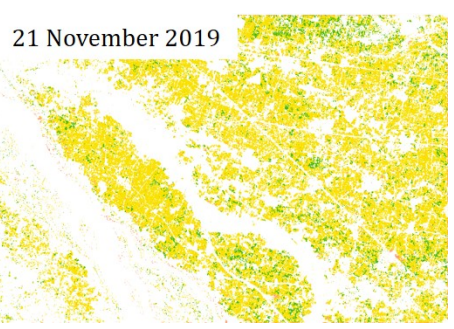

(f) 21 November 2019

Bare field Early tillering

Figure 11. Variation of cluster using MF3CD scattering power components for the rice growing season with in the extent marked in yellow colour in Figure 1 
Table 1. Radarsat-2 data acquired for the Indian test site

\begin{tabular}{|c|c|c|c|c|}
\hline Acquisition date & Beam mode & Incidence angle range $\left(^{\circ}\right)$ & Orbit & azimuth $(\mathrm{m}) \times$ range $(\mathrm{m})$ \\
\hline 06 June 2019 & \multirow{6}{*}{ FQ15W } & $33.73-36.65$ & \multirow{6}{*}{ Ascending } & \multirow{6}{*}{$4.73 \times 5.11$} \\
\hline 24 July 2019 & & $33.73-36.65$ & & \\
\hline 17 August 2019 & & $33.73-36.65$ & & \\
\hline 10 September 2019 & & $33.73-36.65$ & & \\
\hline 04 October 2019 & & $33.73-36.64$ & & \\
\hline 21 November 2019 & & $33.73-36.64$ & & \\
\hline
\end{tabular}


Table 2. Description of rice morphology at different growth stages

\begin{tabular}{|c|c|c|}
\hline Date & Growth stage & Description \\
\hline 06 June 2019 & Bare field (BF) & Complete soil layer is exposed with no standing crop \\
\hline 24 July 2019 & Early tillering (ET) & 1 to 3 tillers detectable \\
\hline 17 August 2019 & Advanced tillering (AT) & Maximum number of tillers detectable \\
\hline 10 September 2019 & Booting $(\mathrm{B})$ & Flag leaf sheath swollen \\
\hline 04 October 2019 & Flowering $(\mathrm{F})$ & Anthers visible on most spikelets \\
\hline 21 November 2019 & Maturity (M) & Grain becomes hard and plants appear yellowish \\
\hline
\end{tabular}


Table 3. Statistics (mean \pm standard deviation) of bio-physical and soil parameters at different phenology stages of rice. Here, PH: plant height, PAI: plant area index, SM: soil moisture and NA: No measurements available

\begin{tabular}{|c|c|c|c|c|}
\hline Date & $\mathbf{P H}(\mathrm{cm})$ & PAI $\left(\mathrm{m}^{2} \mathrm{~m}^{-2}\right)$ & SM $(\%)$ & Growth stage \\
\hline 06 June 2019 & NA & NA & $31.92 \pm 6.10$ & Bare field \\
\hline 24 July 2019 & $22.30 \pm 3.21$ & $0.60 \pm 0.10$ & Saturated & Early tillering \\
\hline 17 August 2019 & $49.26 \pm 7.12$ & $1.86 \pm 0.36$ & Saturated & $\begin{array}{l}\text { Advanced } \\
\text { tillering }\end{array}$ \\
\hline 10 September 2019 & $96.16 \pm 8.76$ & $6.03 \pm 0.80$ & Saturated & Booting \\
\hline 04 October 2019 & $98.93 \pm 4.76$ & $6.16 \pm 0.13$ & $44.60 \pm 0.72$ & Flowering \\
\hline 21 November 2019 & $99.32 \pm 1.82$ & $5.86 \pm 0.62$ & $41.16 \pm 8.04$ & Maturity \\
\hline
\end{tabular}


Table 4. Producer's and User's accuracy of phenology stages of rice for MF3CF, An3D, F3D and Y3D decomposed power components using a RF classifier. BF: Bare field, ET: Early tillering, AT: Advanced tillering, B: Booting, F: Flowering, M: Maturity, PA: Producer's accuracy, UA: User's accuracy

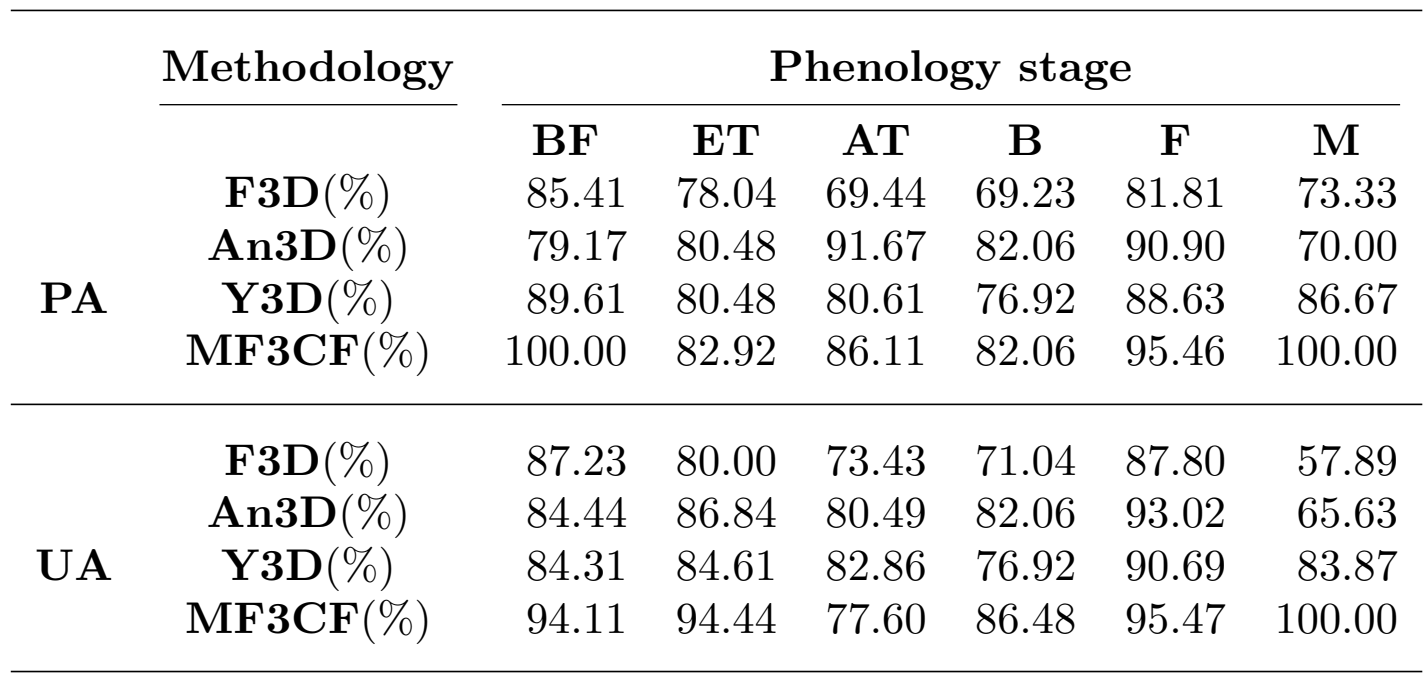


Table 5. Global Measures for FP decomposition techniques

\begin{tabular}{lccc}
\hline Method & Overall accuracy $(\%)$ & $\kappa$ & $p$-value \\
\hline F3D & 76.89 & 0.73 & $1.76 \times 10^{-7}$ \\
An3D & 82.77 & 0.82 & $2.92 \times 10^{-7}$ \\
Y3D & 84.03 & 0.81 & $2.83 \times 10^{-8}$ \\
MF3CF & 91.17 & 0.91 & $1.84 \times 10^{-8}$ \\
\hline
\end{tabular}


Table 6. Producer's and User's accuracy of phenology stages of rice for MF3CD, U2D and T $\mathbf{T}_{2}$ matrix elements using a RF classifier. BF: Bare field, ET: Early tillering, AT: Advanced tillering, B: Booting, F: Flowering, M: Maturity, PA: Producer's accuracy, UA: User's accuracy. The results are separately compared with MF3CF.

\begin{tabular}{|c|c|c|c|c|c|c|c|}
\hline & \multirow[t]{2}{*}{ Methodology } & \multicolumn{6}{|c|}{ Phenology stage } \\
\hline & & BF & ET & AT & B & $\mathbf{F}$ & M \\
\hline & $\mathbf{T}_{2}$ Elements(\%) & 82.92 & 79.48 & 59.61 & 57.60 & 82.06 & 64.86 \\
\hline & $\mathbf{U} 2 \mathbf{D}(\%)$ & 87.80 & 84.62 & 64.28 & 65.00 & 82.04 & 75.67 \\
\hline \multirow[t]{4}{*}{ PA } & $\operatorname{MF3CD}(\%)$ & 95.83 & 80.48 & 83.33 & 89.47 & 90.90 & 100.00 \\
\hline & $\operatorname{MF3CF}(\%)$ & 100.00 & 82.92 & 86.11 & 82.06 & 95.46 & 100.00 \\
\hline & $\mathbf{T}_{2}$ Elements(\%) & 97.14 & 75.61 & 80.64 & 52.27 & 91.43 & 46.16 \\
\hline & $\mathbf{U} 2 \mathbf{D}(\%)$ & 97.29 & 80.49 & 81.82 & 63.41 & 91.43 & 54.90 \\
\hline \multirow[t]{2}{*}{ UA } & MF3CD $(\%)$ & 95.83 & 91.67 & 83.33 & 87.18 & 93.02 & 70.60 \\
\hline & MF3CF $(\%)$ & 94.11 & 94.44 & 77.60 & 86.48 & 95.47 & 100.00 \\
\hline
\end{tabular}


Table 7. Global Measures for DP decomposition techniques

\begin{tabular}{lccc}
\hline \multicolumn{1}{c}{ Method } & Overall accuracy $(\%)$ & $\kappa$ & $p$-value \\
\hline T $_{2}$ Elements & 71.00 & 0.66 & $3.93 \times 10^{-8}$ \\
U2D & 76.47 & 0.79 & $2.76 \times 10^{-8}$ \\
MF3CD & 86.97 & 0.84 & $1.93 \times 10^{-8}$ \\
\hline MF3CF & 91.17 & 0.91 & $1.84 \times 10^{-8}$ \\
\hline
\end{tabular}

